\title{
Sobolev Lifting over Invariants
}

Adam PARUSIŃSKI ${ }^{\mathrm{a}}$ and Armin RAINER $\mathrm{b}$

a) Université Côte d'Azur, CNRS, LJAD, UMR 7351, 06108 Nice, France

E-mail: adam.parusinski@univ-cotedazur.fr

b) Fakultät für Mathematik, Universität Wien, Oskar-Morgenstern-Platz 1, A-1090 Wien, Austria

E-mail: armin.rainer@univie.ac.at

Received November 04, 2020, in final form March 29, 2021; Published online April 10, 2021

https://doi.org/10.3842/SIGMA.2021.037

\begin{abstract}
We prove lifting theorems for complex representations $V$ of finite groups $G$. Let $\sigma=\left(\sigma_{1}, \ldots, \sigma_{n}\right)$ be a minimal system of homogeneous basic invariants and let $d$ be their maximal degree. We prove that any continuous map $\bar{f}: \mathbb{R}^{m} \rightarrow V$ such that $f=\sigma \circ \bar{f}$ is of class $C^{d-1,1}$ is locally of Sobolev class $W^{1, p}$ for all $1 \leq p<d /(d-1)$. In the case $m=1$ there always exists a continuous choice $\bar{f}$ for given $f: \mathbb{R} \rightarrow \sigma(V) \subseteq \mathbb{C}^{n}$. We give uniform bounds for the $W^{1, p}$-norm of $\bar{f}$ in terms of the $C^{d-1,1}$-norm of $f$. The result is optimal: in general a lifting $\bar{f}$ cannot have a higher Sobolev regularity and it even might not have bounded variation if $f$ is in a larger Hölder class.
\end{abstract}

Key words: Sobolev lifting over invariants; complex representations of finite groups; $Q-$ valued Sobolev functions

2020 Mathematics Subject Classification: 22E45; 26A16; 46E35; 14L24

\section{Introduction}

\subsection{Motivation and introduction to the problem}

This paper arose from our wish to understand and extend the principles behind our proof of the optimal Sobolev regularity of roots of smooth families of polynomials [13, 15, 16, 17]. Here we look at this problem from a representation theoretic view point. In fact, choosing the roots of a family of monic polynomials

$$
P_{a(x)}(Z)=Z^{n}+\sum_{j=1}^{n} a_{j}(x) Z^{n-j}
$$

means solving the system of equations

$$
\begin{aligned}
& a_{1}(x)=\sum_{j=1}^{n} \lambda_{j}(x), \\
& a_{2}(x)=\sum_{1 \leq j_{1}<j_{2} \leq n} \lambda_{j_{1}}(x) \lambda_{j_{2}}(x), \\
& \ldots \ldots \ldots \ldots \ldots \ldots \ldots \ldots \ldots \ldots \ldots \ldots \ldots \ldots \ldots \\
& a_{n}(x)=\prod_{j=1}^{n} \lambda_{j}(x)
\end{aligned}
$$

for functions $\lambda_{j}, j=1, \ldots, n$. In other words, it means lifting the map $a=\left(a_{1}, \ldots, a_{n}\right)$ over the map $\sigma=\left(\sigma_{1}, \ldots, \sigma_{n}\right)$ the components of which are the elementary symmetric functions in $n$ variables,

$$
\sigma_{i}\left(X_{1}, \ldots, X_{n}\right)=\sum_{1 \leq j_{1}<\cdots<j_{i} \leq n} X_{j_{1}} X_{j_{2}} \cdots X_{j_{i}}
$$


The map $\sigma$ can be identified with the orbit projection of the tautological representation of the symmetric group $\mathrm{S}_{n}$ on $\mathbb{C}^{n}$ (it acts by permuting the coordinates).

In this paper we shall solve the generalized problem for complex finite-dimensional representations of finite groups. Let $G$ be a finite group. Let $\rho: G \rightarrow \operatorname{GL}(V)$ be a representation of $G$ on a finite-dimensional complex vector space $V$. By Hilbert's finiteness theorem the algebra of invariant polynomials $\mathbb{C}[V]^{G}$ is finitely generated. Let $\sigma_{1}, \ldots, \sigma_{n}$ be a system of generators, we call them basic invariants, and let $\sigma=\left(\sigma_{1}, \ldots, \sigma_{n}\right)$ be the resulting map $\sigma: V \rightarrow \mathbb{C}^{n}$. The map $\sigma$ separates $G$-orbits and hence induces a homeomorphism between the orbit space $V / G$ and the image $\sigma(V)$. (Notice that since $G$ is finite and thus all $G$-orbits are closed, there is a bijection between the orbits and the points in the affine variety $V / / G$ with coordinate ring $\mathbb{C}[V]^{G}$; in other words the categorical quotient $V / / G$ is a geometric quotient.) As a consequence we may identify $V / G$ with $\sigma(V)$ and the canonical orbit projection $V \rightarrow V / G$ with $\sigma: V \rightarrow \sigma(V)$. We will also write $G \circlearrowleft V$ for the representation $\rho$.

The basic invariants can be chosen to be homogeneous polynomials. A system of homogeneous basic invariants is minimal if none among them is superfluous. In that case their number and their degrees are uniquely determined (cf. [5, p. 95]).

Assume that a map $f: \Omega \rightarrow \sigma(V)$ defined on some open subset $\Omega \subseteq \mathbb{R}^{m}$ is given. We will assume that $f$ possesses some degree of differentiability as a map into $\mathbb{C}^{n}$. The question we will address in this paper is the following:

How differentiable can lifts of $f$ over $\sigma$ be? By a lift of $f$ over $\sigma$ we mean a map $\bar{f}: \Omega \rightarrow V$ such that $f=\sigma \circ \bar{f}$.

Simple examples show that, in general, a big loss of regularity occurs from $f$ to lifts of $f$. We will determine the optimal regularity of lifts among the Sobolev spaces $W^{1, p}$ under minimal differentiability requirements on $f$. In particular, the optimal $p>1$ will be determined as an explicit function of the maximal homogeneity degree of the basic invariants.

Note that the results do not depend on the choice of the basic invariants since any two choices differ by a polynomial diffeomorphism.

Our results could be useful in connection with the orbit space reduction of equivariant dynamical system for lifting the solutions from orbit space (even though it is not clear when a lifted solution solves the original differential equation). Another application to multi-valued Sobolev functions is discussed at the end of the paper.

\subsection{The main results}

The first result concerns the lifting of curves. We recall that, since $G$ is finite, each continuous $a: I \rightarrow \sigma(V)$, where $I \subseteq \mathbb{R}$ is an interval, has a continuous lift $\bar{a}: I \rightarrow V$, by [9, Theorem 5.1].

Theorem 1.1. Let $G$ be a finite group and let $G \circlearrowleft V$ be a representation of $G$ on a finitedimensional complex vector space $V$. Let $\sigma=\left(\sigma_{1}, \ldots, \sigma_{n}\right)$ be a (minimal) system of homogeneous basic invariants of degrees $d_{1}, \ldots, d_{n}$ and set $d=\max _{i} d_{i}$. Let $a \in C^{d-1,1}([\alpha, \beta], \sigma(V))$ be a curve defined on an open bounded interval $(\alpha, \beta)$ with values in $\sigma(V)$. Then each continuous lift $\bar{a}:(\alpha, \beta) \rightarrow V$ of a over $\sigma$ is absolutely continuous and belongs to $W^{1, p}((\alpha, \beta), V)$ with

$$
\left\|\bar{a}^{\prime}\right\|_{L^{p}((\alpha, \beta))} \leq C(G \circlearrowleft V,(\beta-\alpha), p) \max _{1 \leq j \leq n}\left\|a_{j}\right\|_{C^{d-1,1}([\alpha, \beta])}^{1 / d_{j}}
$$

for all $1 \leq p<d /(d-1)$, where $C$ is a constant which depends only on the representation $G \circlearrowleft V$, the length of the interval $(\alpha, \beta)$, and $p$. 
The conclusion of the theorem is in general optimal among Sobolev spaces, the differentiability assumption on $a$ is best possible; see Remark 3.2. Here and below we use the notation

$$
C^{d-1,1}([\alpha, \beta], \sigma(V)):=C^{d-1,1}\left([\alpha, \beta], \mathbb{C}^{n}\right) \cap \sigma(V)^{(\alpha, \beta)},
$$

the Hölder class $C^{d-1,1}$ is defined in Section 2.

\section{Remark 1.2.}

(a) In general the constant in (1.1) is of the form

$$
C(G \circlearrowleft V, p) \max \left\{1,(\beta-\alpha)^{1 / p},(\beta-\alpha)^{-1+1 / p}\right\} .
$$

(b) If the curve $a$ starts, ends, or passes through 0 (that is the most singular point in $\sigma(V)$ ), then the constant in (1.1) is of the form

$$
C(G \circlearrowleft V, p) \max \left\{1,(\beta-\alpha)^{1 / p}\right\} .
$$

(c) If the representation is coregular, then for all $a$ satisfying the assumptions of Theorem 1.1 the constant is of the form (1.2). A representation $G \circlearrowleft V$ is called coregular if $\mathbb{C}[V]^{G}$ is isomorphic to a polynomial algebra, i.e., there is a system of basic invariants without polynomial relations among them. By the Shephard-Todd-Chevalley theorem [2, 19, 20], this is the case if and only if $G$ is generated by pseudoreflections.

(d) The constant is also of the form (1.2) if the curve $a$ satisfies $a^{(j)}(\alpha)=a^{(j)}(\beta)=0$ for all $j=1, \ldots, d-1$.

Question 1.3. The constant in (1.1) tends to infinity as $p \rightarrow d /(d-1)=: d^{\prime}$. Our proof yields that it blows up like a power of $\left(d^{\prime}-p\right)^{-1 / p}$, since we have to iterate the inequality (2.1) several times when we pass from $L_{w}^{d^{\prime}}$-(quasi)norm to $L^{p}$-norm. This is necessary, since the former is not $\sigma$-additive. We expect that the asymptotic behavior of the constant as $p \rightarrow d^{\prime}$ is actually better: Is the constant actually $O\left(\left(d^{\prime}-p\right)^{-1 / p}\right)$ as $p \rightarrow d^{\prime}$ ? Can one replace the $L^{p}$-norm of $\bar{a}^{\prime}$ by the $L_{w}^{d^{\prime}}$-(quasi)norm in (1.1)?

The lifting of mappings defined in open domains of dimension $m>1$ essentially admits the same regularity as for curves, provided that continuous lifting is possible. However, there are well-known topological obstructions for continuous lifting in general. We will prove the following

Theorem 1.4. In the setting of Theorem 1.1 let $f \in C^{d-1,1}(\bar{\Omega}, \sigma(V))$, where $\Omega \subseteq \mathbb{R}^{m}$ is an open bounded box $\Omega=I_{1} \times \cdots \times I_{m}$. Then each continuous lift $\bar{f}: U \rightarrow V$ of $f$ over $\sigma$ defined on an open subset $U \subseteq \Omega$ belongs to $W^{1, p}(U, V)$ for all $1 \leq p<d /(d-1)$ and satisfies

$$
\|\nabla \bar{f}\|_{L^{p}(U)} \leq C(G \circlearrowleft V, \Omega, m, p) \max _{1 \leq j \leq n}\left\|f_{j}\right\|_{C^{d-1,1}(\bar{\Omega})}^{1 / d_{j}}
$$

for all $1 \leq p<d /(d-1)$, where $C$ is a constant which depends only on the representation $G \circlearrowleft V$, on $\Omega, m$, and $p$.

The case $U=\Omega$ is not excluded! It is clear that Theorem 1.4 implies a version of the statement, where $\Omega \subseteq \mathbb{R}^{m}$ is any bounded open set, $U \Subset \Omega$ is relatively compact open in $\Omega$, and the constant also depends on $U$ (or more precisely on a cover of $U$ by boxes contained in $\Omega$ ). Concerning a global result we have the following

Remark 1.5. If $G \circlearrowleft V$ is coregular, then Theorem 1.4 holds as stated for any bounded Lipschitz domain $\Omega$.

When continuous lifting is impossible, we expect that a general $B V$-lifting result is true analogous to the existence of $B V$-roots for smooth polynomials proved in [17]. We shall not pursue that question in this paper. 


\subsection{Linearly reductive groups}

An algebraic group $G$ is called linearly reductive if for each rational representation $V$ and each subrepresentation $W \subseteq V$ there is a subrepresentation $W^{\prime} \subseteq V$ such that $V=W \oplus W^{\prime}$.

For rational representations of linearly reductive groups $G$ Hilbert's finiteness theorem is true, that is the algebra of $G$-invariant polynomials $\mathbb{C}[V]^{G}$ is finitely generated. Let $\sigma=\left(\sigma_{1}, \ldots, \sigma_{n}\right)$ be a system of generators. Then the map $\sigma: V \rightarrow \sigma(V) \subseteq \mathbb{C}^{n}$ can be identified with the morphism $V \rightarrow V / / G$ induced by the inclusion $\mathbb{C}[V]^{G} \rightarrow \mathbb{C}[V]$; the categorical quotient $V / / G$ is the affine variety with coordinate ring $\mathbb{C}[V]^{G}$. In general $V / / G$ is not a geometric quotient, that is the $G$-orbits in $V$ are not in a one-to-one correspondence with the points in $V / / G$. In fact, for every point $z \in V / / G$ there is a unique closed orbit in the fiber $\sigma^{-1}(z)$ which lies in the closure of every other orbit in this fiber.

In this setting it is not clear if a continuous curve in $\sigma(V)$ admits a continuous lift to $V$. The notion of stability in geometric invariant theory provides a remedy. A point $v \in V$ is called stable if the orbit $G v$ is closed and the isotropy group $G_{v}=\{g \in G: g v=v\}$ is finite. The set $V^{s}$ of stable points in $V$ is $G$-invariant and open in $V$, and its image $\sigma\left(V^{s}\right)$ is open in $V / / G \cong \sigma(V)$ (cf. [11, Proposition 5.15]). The restriction $\sigma: V^{s} \rightarrow \sigma\left(V^{s}\right)$ of the map $\sigma$ provides a one-to-one correspondence between points in $\sigma\left(V^{s}\right) \cong V^{s} / G$ and $G$-orbits in $V^{s}$, that is $V^{s} / G$ is a geometric quotient.

Lemma 1.6. Let $a: I \rightarrow \sigma\left(V^{s}\right)$, where $I \subseteq \mathbb{R}$ is an open interval, be continuous. Then a has a continuous lift $\bar{a}: I \rightarrow V^{s}$.

Proof. For every $v \in \sigma^{-1}(a(I))$ there is a local continuous lift $\bar{a}_{v}$ of $a$ defined on some open subinterval $I_{v}$ of $I$ with $\bar{a}_{v}\left(t_{v}\right)=v$ for some point $t_{v} \in I_{v}$. This follows from the lifting theorem [9, Theorem 5.1], since locally at any $v$ the problem can be reduced to the slice representation of the isotropy group $G_{v}$ which is finite (cf. Theorem 4.2). Now each continuous lift $\bar{a}$ of $a$ defined on a proper subinterval $J$ of $I$ has an extension to a larger interval $J^{\prime} \subseteq I$. Thus there is a continuous lift on $I$. Indeed, say the right endpoint $t_{1}$ of $J$ lies in $I$. There is continuous lift $\bar{a}_{v}: I_{v} \rightarrow V^{s}$ for $v \in \sigma^{-1}\left(a\left(t_{1}\right)\right)$. Choose $t_{0} \in J \cap I_{v}$ and $g \in G$ such that $\bar{a}\left(t_{0}\right)=g \bar{a}_{v}\left(t_{0}\right)$. Then $g \bar{a}_{v}$ extends the continuous lift $\bar{a}$ beyond $t_{1}$.

As a corollary of Theorem 1.1 we obtain

Theorem 1.7. Let $G$ be a linearly reductive group and let $G \circlearrowleft V$ be a rational representation of $G$ on a finite-dimensional complex vector space $V$. Let $\sigma=\left(\sigma_{1}, \ldots, \sigma_{n}\right)$ be a (minimal) system of homogeneous basic invariants of degrees $d_{1}, \ldots, d_{n}$ and set $d=\max _{i} d_{i}$. Let $a \in$ $C^{d-1,1}\left([\alpha, \beta], \sigma\left(V^{s}\right)\right)$ be a curve defined on a compact interval with $a([\alpha, \beta]) \subseteq \sigma\left(V^{s}\right)$. Then there exists an absolutely continuous lift $\bar{a}:[\alpha, \beta] \rightarrow V^{s}$ of a over $\sigma$ which belongs to $W^{1, p}\left([\alpha, \beta], V^{s}\right)$ with

$$
\left\|\bar{a}^{\prime}\right\|_{L^{p}([\alpha, \beta])} \leq C(G \circlearrowleft V,[\alpha, \beta], p) \max _{1 \leq j \leq n}\left\|a_{j}\right\|_{C^{d-1,1}([\alpha, \beta])}^{1 / d_{j}}
$$

for all $1 \leq p<d /(d-1)$.

Proof. Since the lifting problem can be reduced to the slice representations (cf. Theorem 4.2 and Lemma 4.5), and for all $v \in V^{s}$ the isotropy group $G_{v}$ is finite, Theorem 1.1 implies that for all $v \in \sigma^{-1}\left(a([\alpha, \beta])\right.$ there exists a local absolutely continuous lift $\bar{a}_{v}$ of $a$ defined on a subinterval $I_{v}$ of $[\alpha, \beta]$ which is open in the relative topology on $[\alpha, \beta]$ such that

$$
\left\|\bar{a}_{v}^{\prime}\right\|_{L^{p}\left(I_{v}\right)} \leq C\left(G \circlearrowleft V,\left|I_{v}\right|, p\right) \max _{1 \leq j \leq n}\left\|a_{j}\right\|_{C^{d-1,1}\left(\bar{I}_{v}\right)}^{1 / d_{j}}, \quad 1 \leq p<\frac{d}{d-1},
$$


and there is a point $t_{v} \in I_{v}$ with $\bar{a}_{v}\left(t_{v}\right)=v$. By compactness, there is a finite collection of local lifts which cover $[\alpha, \beta]$. It is then easy to glue these pieces (after applying fixed transformations from $G$ ) to an absolutely continuous lift $\bar{a}$ defined on $[\alpha, \beta]$ and satisfying (1.4).

For a mapping $f$ defined on a compact subset $K$ of $\mathbb{R}^{m}$ with $f(K) \subseteq \sigma\left(V^{s}\right)$ the situation is more complicated. We can apply Theorem 1.4 to the slice representations at any point $v \in V^{s}$. But it is not clear if these local (and partial) lifts can be glued together in a continuous fashion.

\subsection{Polar representations}

More can be said for polar representations (which include e.g. the adjoint actions). The following results can be found in [3]. Let $G$ be a linearly reductive group and let $G \circlearrowleft V$ be a representation of $G$ on a finite-dimensional complex vector space $V$. Let $v \in V$ be such that $G v$ is closed and consider the linear subspace $\Sigma_{v}=\{x \in V: \mathfrak{g} x \subseteq \mathfrak{g} v\}$, where $\mathfrak{g}$ denotes the Lie algebra of $G$. All orbits that intersect $\Sigma_{v}$ are closed, whence $\operatorname{dim} \Sigma_{v} \leq \operatorname{dim} V / / G$. The representation $G \circlearrowleft V$ is said to be polar if there exists $v \in V$ with closed orbit $G v$ and $\operatorname{dim} \Sigma_{v}=\operatorname{dim} V / / G$. Then $\Sigma_{v}$ is called a Cartan subspace of $V$. Any two Cartan subspaces are $G$-conjugate. Let us fix one Cartan space $\Sigma$. All closed orbits in $V$ intersect $\Sigma$.

The Weyl group $W$ is defined by $W=N_{G}(\Sigma) / Z_{G}(\Sigma)$, where $N_{G}(\Sigma)=\{g \in G: g \Sigma=\Sigma\}$ is the normalizer and $Z_{G}(\Sigma)=\{g \in G: g x=x$ for all $x \in \Sigma\}$ is the centralizer of $\Sigma$ in $G$. The Weyl group is finite and the intersection of any closed $G$-orbit in $V$ with the Cartan subspace is precisely one $W$-orbit. The ring $\mathbb{C}[V]^{G}$ is isomorphic via restriction to the ring $\mathbb{C}[\Sigma]^{W}$. If $G$ is connected, then $W$ is a pseudoreflection group and hence $\mathbb{C}[V]^{G} \cong \mathbb{C}[\Sigma]^{W}$ is a polynomial ring, by the Shephard-Todd-Chevalley theorem [2, 19, 20].

Theorem 1.8. Let $G \circlearrowleft V$ be a polar representation of a linearly reductive group $G$. Let $\sigma=\left(\sigma_{1}, \ldots, \sigma_{n}\right)$ be a (minimal) system of homogeneous basic invariants of degrees $d_{1}, \ldots, d_{n}$ and set $d=\max _{i} d_{i}$.

1. Let $a \in C^{d-1,1}([\alpha, \beta], \sigma(V))$ be a curve defined on an open bounded interval $(\alpha, \beta)$ with values in $\sigma(V)$. Then there exists an absolutely continuous lift $\bar{a}:(\alpha, \beta) \rightarrow V$ of a over $\sigma$ which belongs to $W^{1, p}((\alpha, \beta), V)$ for all $1 \leq p<d /(d-1)$ and satisfies (1.1).

2. Let $f \in C^{d-1,1}(\bar{\Omega}, \sigma(V))$, where $\Omega \subseteq \mathbb{R}^{m}$ is an open bounded box $\Omega=I_{1} \times \cdots \times I_{m}$. Each continuous lift $\bar{f}$ defined in an open subset $U \subseteq \Omega$ with values in a Cartan subspace $\Sigma$ is of class $W^{1, p}$ on $U$ for all $1 \leq p<d /(d-1)$ and satisfies (1.3).

3. In the case that $G$ is connected the constant in (1.1) is of the form (1.2) and $\Omega$ can be any bounded Lipschitz domain.

Proof. Apply Theorems 1.1 and 1.4 to the Weyl group $W$ acting on a Cartan subspace $\Sigma$. If $G$ is connected, then $W \circlearrowleft \Sigma$ is coregular, so (3) follows from Remarks 1.2 and 1.5.

\subsection{A related problem}

In an analogous way one may consider the case that $V$ is a real finite-dimensional vector space and $\rho: G \rightarrow \mathrm{O}(V)$ is an orthogonal representation of a finite group. Again the algebra of $G$ invariant polynomials $\mathbb{R}[V]^{G}$ is finitely generated, and a system of basic invariants $\sigma$ allows us to identify $\sigma(V)$ with the orbit space $V / G$. In this case $\sigma(V)$ is a semialgebraic subset of $\mathbb{R}^{n}$. In that setting the problem was solved in [14]:

Theorem 1.9. Let $G$ be a finite group and let $G \circlearrowleft V$ be an orthogonal representation of $G$ on a finite-dimensional real vector space $V$. Let $\sigma=\left(\sigma_{1}, \ldots, \sigma_{n}\right)$ be a (minimal) system of homogeneous basic invariants of degrees $d_{1}, \ldots, d_{n}$ and set $d=\max _{i} d_{i}$. 
1. Let $a \in C^{d-1,1}([\alpha, \beta], \sigma(V))$. Then each continuous lift $\bar{a}:(\alpha, \beta) \rightarrow V$ of a over $\sigma$ belongs to $W^{1, \infty}((\alpha, \beta), V)$ with

$$
\left\|\bar{a}^{\prime}\right\|_{L^{\infty}((\alpha, \beta))} \leq C(G \circlearrowleft V,(\beta-\alpha)) \max _{1 \leq j \leq n}\left\|a_{j}\right\|_{C^{d-1,1}([\alpha, \beta])}^{1 / d_{j}} .
$$

Every continuous curve in $\sigma(V)$ has a continuous lift.

2. Let $f \in C^{d-1,1}(\bar{\Omega}, \sigma(V))$, where $\Omega \subseteq \mathbb{R}^{m}$ is open and bounded. Then each continuous lift $\bar{f}: U \rightarrow V$ of $f$ over $\sigma$ defined on an open subset $U \subseteq \Omega$ belongs to $W^{1, \infty}(U, V)$ with

$$
\|\nabla \bar{f}\|_{L^{\infty}(U)} \leq C(G \circlearrowleft V, \Omega, U, m) \max _{1 \leq j \leq n}\left\|a_{j}\right\|_{C^{d-1,1}(\bar{\Omega})}^{1 / d_{j}}
$$

In the special case of the tautological representation of $S_{n}$ on $\mathbb{R}^{n}$ this corresponds to the problem of choosing the roots of hyperbolic polynomials, i.e., monic polynomials all roots of which are real; see [13].

The main difference between the complex and the real problem is that in the latter case the map $v \mapsto\langle v, v\rangle=\|v\|^{2}$ is an invariant polynomial which may be taken without loss of generality as a basic invariant and thus as a component of the map $\sigma$. The key is that this basic invariant dominates all the others, by homogeneity,

$$
\left|\sigma_{j}(v)\right| \leq \max _{\|w\|=1}\left|\sigma_{j}(w)\right|\|v\|^{d_{j}}
$$

Even though we can always choose an invariant Hermitian inner product in the complex case (by averaging over $G$ ) and hence assume that the representation is unitary, the invariant form $v \mapsto\|v\|^{2}$ is not a member of $\mathbb{C}[V]^{G}$. The fact that there is no invariant that dominates all others makes the complex case much more difficult.

\subsection{Elements of the proof}

We briefly describe the strategy of the proof of Theorem 1.1.

The basic building block of the proof is that the result holds for finite rotation groups $C_{d}$ in $\mathbb{C}$, where $\mathbb{C}[\mathbb{C}]^{C_{d}}$ is generated by $z \mapsto z^{d}$ and a lift of a map $f$ is a solution of the equation $z^{d}=f$. This follows from [6]. Among all representations of finite groups $G$ of order $|G|$ it is the one with the worst loss of regularity, since in general $d \leq|G|$, by Noether's degree bound, and equality can only happen for cyclic groups (see Section 3).

In the general case we first observe that evidently one may reduce to the case that the linear subspace $V^{G}$ of invariant vectors is trivial. Then Luna's slice theorem (see Theorem 4.2) allows us to reduce the problem locally to the slice representation $G_{v} \circlearrowleft N_{v}$ of the isotropy group $G_{v}=\{g \in G: g v=v\}$ on $N_{v}$, where $T_{v} V \cong T_{v}(G v) \oplus N_{v}$ is a $G_{v}$-splitting. Since in our case $G$ is finite, we have $N_{v} \cong V$. The assumption $V^{G}=\{0\}$ entails that for all $v \in V \backslash\{0\}$ the isotropy group $G_{v}$ is a proper subgroup of $G$ which suggests to use induction.

For this induction scheme to work we need that the slice reduction is uniform in the sense that it does not depend on the parameter $t$ of the curve $a$ in $\sigma(V) \subseteq \mathbb{C}^{n}$. We achieve this by considering the curve

$$
\underline{a}=\left(a_{k}^{-d_{1} / d_{k}} a_{1}, \ldots, a_{k}^{-d_{n} / d_{k}} a_{n}\right), \quad \text { when } \quad a_{k} \neq 0,
$$

and the compactness of the set of all $\underline{a} \in \sigma(V)$ such that $\left|\underline{a}_{j}\right| \leq 1$ for all $j=1, \ldots, n$ and $\underline{a}_{k}=1$. Let us emphasize that hereby we use a fixed continuous selection $\hat{a}_{k}$ of the multi-valued function $a_{k}^{1 / d_{k}}$ which is absolutely continuous by the result for the rotation group $C_{d_{k}} \circlearrowleft \mathbb{C}$. 
If $a \in C^{d-1,1}([\alpha, \beta], \sigma(V))$ and $t_{0} \in(\alpha, \beta)$ is such that $a\left(t_{0}\right) \neq 0$, then we choose $k \in\{1, \ldots, n\}$ dominant in the sense that

$$
\left|a_{k}^{1 / d_{k}}\left(t_{0}\right)\right|=\max _{1 \leq j \leq n}\left|a_{j}^{1 / d_{j}}\left(t_{0}\right)\right| \neq 0 .
$$

It is easy to extend the lifts to the points, where $a$ vanishes, so we will not discuss them here. We work on a small interval $I$ containing $t_{0}$ such that for all $j=1, \ldots, n$ and $s=1, \ldots, d-1$,

$$
\begin{aligned}
& \left\|a_{j}^{(s)}\right\|_{L^{\infty}(I)} \leq C(d)|I|^{-s}\left|a_{k}\left(t_{0}\right)\right|^{d_{j} / d_{k}}, \\
& \operatorname{Lip}_{I}\left(a_{j}^{(d-1)}\right) \leq C(d)|I|^{-d}\left|a_{k}\left(t_{0}\right)\right|^{d_{j} / d_{k}} .
\end{aligned}
$$

This can be achieved by choosing the interval $I$ in such a way that $t_{0} \in I \subseteq(\alpha, \beta)$ and

$$
M|I|+\sum_{j=1}^{n}\left\|\left(a_{j}^{1 / d_{j}}\right)^{\prime}\right\|_{L^{1}(I)} \leq B\left|a_{k}\left(t_{0}\right)\right|^{1 / d_{k}}
$$

where $B$ is a suitable constant which depends only on the representation and the constant $M$ depends on the representation and the curve $a$. Notice that here we use again absolute continuity of radicals (i.e., the result for complex rotation groups). Uniform slice reduction allows us to switch to a reduced curve $b: I \rightarrow \tau(W)$ of class $C^{d-1,1}$, where $H \circlearrowleft W$ is a slice representation of $G \circlearrowleft V$ and the map $\tau=\left(\tau_{1}, \ldots, \tau_{m}\right)$ consists of a system of homogeneous generators for $\mathbb{C}[W]^{H}$. For convenience we will refer to the tuple $\left(a, I, t_{0}, k ; b\right)$ as reduced admissible data for $G \circlearrowleft V$.

The core of the proof (see Proposition 8.2) is to show that, if $\left(a, I, t_{0}, k ; b\right)$ is reduced admissible data for $G \circlearrowleft V$, then every continuous lift $\bar{b}: I \rightarrow W$ of $b$ is absolutely continuous and satisfies

$$
\left\|\bar{b}^{\prime}\right\|_{L^{p}(I)} \leq C(d, p)\left(\left\||I|^{-1}\left|a_{k}\left(t_{0}\right)\right|^{1 / d_{k}}\right\|_{L^{p}(I)}+\sum_{i=1}^{m}\left\|\left(b_{i}^{1 / e_{i}}\right)^{\prime}\right\|_{L^{p}(I)}\right)
$$

for all $1 \leq p<d /(d-1)$, where $e_{i}=\operatorname{deg} \tau_{i}$. This is done by induction on the group order and involves showing that the set of points $t$ in $I$, where $b(t) \neq 0$, can be covered by a special countable collection of intervals on which $b$ defines reduced admissible data for $H \circlearrowleft W$. The difficult part is to assure that each point is covered by at most two intervals in the collection (see Proposition 7.1) which is needed for gluing the local $L^{p}$-estimates to a global estimate on $I$. It would suffice that each point lies in no more than a uniform finite number of intervals, but the crucial thing is that the intervals must not be shrunk (see Remark 7.2).

\subsection{An application: $Q$-valued functions}

In Section 10 we explore an interesting connection between invariant theory and the theory of $Q$-valued functions. These are functions with values in the metric space of unordered $Q$ tuples of points in $\mathbb{R}^{n}$ (or $\mathbb{C}^{n}$ ). There is a natural one-to-one correspondence between unordered $Q$-tuples of points in $\mathbb{K}^{n}$ (where $\mathbb{K}$ stands for $\mathbb{R}$ or $\mathbb{C}$ ) and the $n$-fold direct sum of the tautological representation of the symmetric group $S_{Q}$ on $\mathbb{K}^{Q}$. Using the theory of $Q$-valued Sobolev functions rooted in variational calculus, cf. [1] and [4], we will show that our main results entail optimal multi-valued Sobolev lifting theorems. Thanks to the multi-valuedness there are no topological obstructions for continuity.

\section{Function spaces}

In this section we fix notation for function spaces and recall well-known facts. 


\subsection{Hölder spaces}

Let $\Omega \subseteq \mathbb{R}^{n}$ be open and bounded. We denote by $C^{0}(\Omega)$ the space of continuous complex valued functions on $\Omega$. For $k \in \mathbb{N} \cup\{\infty\}$ (and multi-indices $\gamma$ ) we set

$$
\begin{aligned}
& C^{k}(\Omega)=\left\{f \in \mathbb{C}^{\Omega}: \partial^{\gamma} f \in C^{0}(\Omega), 0 \leq|\gamma| \leq k\right\}, \\
& C^{k}(\bar{\Omega})=\left\{f \in C^{k}(\Omega): \partial^{\gamma} f \text { has a continuous extension to } \bar{\Omega}, 0 \leq|\gamma| \leq k\right\} .
\end{aligned}
$$

For $\alpha \in(0,1]$ a function $f: \Omega \rightarrow \mathbb{C}$ belongs to $C^{0, \alpha}(\bar{\Omega})$ if it is $\alpha$-Hölder continuous in $\Omega$, i.e.,

$$
\operatorname{Höld}_{\alpha, \Omega}(f):=\sup _{x, y \in \Omega, x \neq y} \frac{|f(x)-f(y)|}{|x-y|^{\alpha}}<\infty .
$$

If $f$ is Lipschitz, i.e., $f \in C^{0,1}(\bar{\Omega})$, we write $\operatorname{Lip}_{\Omega}(f):=\operatorname{Höld}_{1, \Omega}(f)$. We define

$$
C^{k, \alpha}(\bar{\Omega})=\left\{f \in C^{k}(\bar{\Omega}): \partial^{\gamma} f \in C^{0, \alpha}(\bar{\Omega}),|\gamma| \leq k\right\},
$$

which is a Banach space when provided with the norm

$$
\|f\|_{C^{k, \alpha}(\bar{\Omega})}:=\max _{|\gamma| \leq k} \sup _{x \in \Omega}\left|\partial^{\gamma} f(x)\right|+\max _{|\gamma|=k} \operatorname{Höld}_{\alpha, \Omega}\left(\partial^{\gamma} f\right) .
$$

\subsection{Lebesgue spaces and weak Lebesgue spaces}

Let $\Omega \subseteq \mathbb{R}^{n}$ be open and $1 \leq p \leq \infty$. Then $L^{p}(\Omega)$ is the Lebesgue space with respect to the $n$-dimensional Lebesgue measure $\mathcal{L}^{n}$. For Lebesgue measurable sets $E \subseteq \mathbb{R}^{n}$ we denote by

$$
|E|=\mathcal{L}^{n}(E)
$$

the $n$-dimensional Lebesgue measure of $E$. Let $p^{\prime}:=p /(p-1)$ denote the conjugate exponent of $p$ with the convention $1^{\prime}:=\infty$ and $\infty^{\prime}:=1$.

Let $1 \leq p<\infty$ and let us assume that $\Omega$ is bounded. The weak $L^{p}$-space $L_{w}^{p}(\Omega)$ is the space of all measurable functions $f: \Omega \rightarrow \mathbb{C}$ such that

$$
\|f\|_{p, w, \Omega}:=\sup _{r>0}\left(r|\{x \in \Omega:|f(x)|>r\}|^{1 / p}\right)<\infty .
$$

It will be convenient to normalize:

$$
\begin{aligned}
\|f\|_{L^{p}(\Omega)}^{*} & :=|\Omega|^{-1 / p}\|f\|_{L^{p}(\Omega)}, \\
\|f\|_{p, w, \Omega}^{*} & :=|\Omega|^{-1 / p}\|f\|_{p, w, \Omega} .
\end{aligned}
$$

Note that $\|1\|_{L^{p}(\Omega)}^{*}=\|1\|_{p, w, \Omega}^{*}=1$. For $1 \leq q<p<\infty$ we have (cf. [7, Exercise 1.1.11])

$$
\begin{aligned}
& \|f\|_{L^{q}(\Omega)}^{*} \leq\|f\|_{L^{p}(\Omega)}^{*}, \\
& \|f\|_{q, w, \Omega}^{*} \leq\|f\|_{L^{q}(\Omega)}^{*} \leq\left(\frac{p}{p-q}\right)^{1 / q}\|f\|_{p, w, \Omega}^{*}
\end{aligned}
$$

and hence $L^{p}(\Omega) \subseteq L_{w}^{p}(\Omega) \subseteq L^{q}(\Omega) \subseteq L_{w}^{q}(\Omega)$ with strict inclusions.

We remark that $\|\cdot\|_{p, w, \Omega}$ is only a quasinorm: the triangle inequality fails, but for $f_{j} \in L_{w}^{p}(\Omega)$ we still have

$$
\left\|\sum_{j=1}^{m} f_{j}\right\|_{p, w, \Omega} \leq m \sum_{j=1}^{m}\left\|f_{j}\right\|_{p, w, \Omega} .
$$

There exists a norm equivalent to $\|\cdot\|_{p, w, \Omega}$ which makes $L_{w}^{p}(\Omega)$ into a Banach space if $p>1$.

The $L_{w}^{p}$-quasinorm is $\sigma$-subadditive: if $\Omega=\bigcup \Omega_{j}$ is a countable open cover, then

$$
\|f\|_{p, w, \Omega}^{p} \leq \sum_{j}\|f\|_{p, w, \Omega_{j}}^{p} \quad \text { for every } \quad f \in L_{w}^{p}(\Omega) .
$$

But it is not $\sigma$-additive. 


\subsection{Sobolev spaces}

For $k \in \mathbb{N}$ and $1 \leq p \leq \infty$ we consider the Sobolev space

$$
W^{k, p}(\Omega)=\left\{f \in L^{p}(\Omega): \partial^{\alpha} f \in L^{p}(\Omega), 0 \leq|\alpha| \leq k\right\},
$$

where $\partial^{\alpha} f$ denote distributional derivatives, with the norm

$$
\|f\|_{W^{k, p}(\Omega)}:=\sum_{|\alpha| \leq k}\left\|\partial^{\alpha} f\right\|_{L^{p}(\Omega)} .
$$

On bounded intervals $I \subseteq \mathbb{R}$ the Sobolev space $W^{1,1}(I)$ coincides with the space $A C(I)$ of absolutely continuous functions on $I$ if we identify each $W^{1,1}$-function with its unique continuous representative. Recall that a function $f: \Omega \rightarrow \mathbb{C}$ on an open subset $\Omega \subseteq \mathbb{R}$ is absolutely continuous $(A C)$ if for every $\epsilon>0$ there exists $\delta>0$ such that for every finite collection of non-overlapping intervals $\left(a_{i}, b_{i}\right), i=1, \ldots, n$, with $\left[a_{i}, b_{i}\right] \subseteq \Omega$ we have

$$
\sum_{i=1}^{n}\left|a_{i}-b_{i}\right|<\delta \Longrightarrow \sum_{i=1}^{n}\left|f\left(a_{i}\right)-f\left(b_{i}\right)\right|<\epsilon .
$$

Notice that $W^{1, \infty}(\Omega) \cong C^{0,1}(\bar{\Omega})$ on Lipschitz domains (or more generally quasiconvex domains) $\Omega$.

We shall also use $W_{\text {loc }}^{k, p}, A C_{\text {loc }}$, etc. with the obvious meaning.

\subsection{Vector valued functions}

For our problem we need to consider mappings of Sobolev regularity with values in a finitedimensional complex vector space $V$. Let us fix a basis $v_{1}, \ldots, v_{n}$ of $V$ and hence a linear isomorphism $\varphi: V \rightarrow \mathbb{C}^{n}$. We say that a mapping $f: \Omega \rightarrow V$ is of Sobolev class $W^{k, p}$ if $\varphi \circ f$ is of class $W^{k, p}$. The space $W^{k, p}(\Omega, V)$ of all such mappings does not depend on the choice of the basis of $V$.

For $f=\left(f_{1}, \ldots, f_{n}\right): \Omega \rightarrow \mathbb{C}^{n}$ we set

$$
\|f\|_{W^{k, p}\left(\Omega, \mathbb{C}^{n}\right)}:=\sum_{j=1}^{n}\left\|f_{j}\right\|_{W^{k, p}(\Omega)} .
$$

If $f \in W^{k, p}(\Omega, V), f \neq 0$, and $\varphi, \psi: V \rightarrow \mathbb{C}^{n}$ are two different basis isomorphisms, then

$$
c \leq \frac{\|\varphi \circ f\|_{W^{k, p}\left(\Omega, \mathbb{C}^{n}\right)}}{\|\psi \circ f\|_{W^{k, p}\left(\Omega, \mathbb{C}^{n}\right)}} \leq C
$$

for positive constants $c, C>0$ which depend only on the linear isomorphism $\varphi \circ \psi^{-1}$. We will denote by $\|f\|_{W^{k, p}(\Omega, V)}$ or simply $\|f\|_{W^{k, p}(\Omega)}$ any of the equivalent norms $\|\varphi \circ f\|_{W^{k, p}\left(\Omega, \mathbb{C}^{n}\right)}$.

Now suppose that we have a representation $\rho: G \rightarrow \operatorname{GL}(V)$ of a finite group $G$ on $V$. By fixing a Hermitian inner product on $V$ and averaging it over $G$ we obtain a Hermitian inner product with respect to which the action of $G$ is unitary. We could equivalently define

$$
\|f\|_{W^{k, p}(\Omega)}=\|f\|_{W^{k, p}(\Omega, V)}:=\sum_{|\alpha| \leq k}\left(\int_{\Omega}\left\|\partial^{\alpha} f\right\|^{p} \mathrm{~d} x\right)^{1 / p},
$$

where $\|\cdot\|$ is the norm associated with the $G$-invariant Hermitian inner product. In that case $\|f\|_{W^{k, p}(\Omega, V)}$ is $G$-invariant. 


\subsection{Extension lemma}

The following extension lemma simply follows from the $\mathbb{C}$-valued version proved in [16]. Similar versions can be found in [15, Lemma 2.1] and [6, Lemma 3.2].

Lemma 2.1. Let $V$ be a finite-dimensional vector space. Let $\Omega \subseteq \mathbb{R}$ be open and bounded, let $f: \Omega \rightarrow V$ be continuous, $p \geq 1$, and set $\Omega_{0}:=\{t \in \Omega: f(t) \neq 0\}$. Assume that $\left.f\right|_{\Omega_{0}} \in$ $A C_{\mathrm{loc}}\left(\Omega_{0}, V\right)$ and $\left.f\right|_{\Omega_{0}} ^{\prime} \in L^{p}\left(\Omega_{0}, V\right)$. Then the distributional derivative of $f$ in $\Omega$ is a measurable function $f^{\prime} \in L^{p}(\Omega, V)$ and

$$
\left\|f^{\prime}\right\|_{L^{p}(\Omega, V)}=\left\|\left.f\right|_{\Omega_{0}} ^{\prime}\right\|_{L^{p}\left(\Omega_{0}, V\right)},
$$

where the $L^{p}$-norms are computed with respect to a fixed basis isomorphism.

\section{$3 \quad$ Finite rotation groups in $\mathbb{C}$}

Let $C_{d} \cong \mathbb{Z} / d \mathbb{Z}$ denote the cyclic group of order $d$ and consider its standard action on $\mathbb{C}$ by rotation. Then $\mathbb{C}[\mathbb{C}]^{C_{d}}$ is generated by $\sigma(z)=z^{d}$. A lift over $\sigma$ of a function $f: \Omega \rightarrow \mathbb{C}$ is a solution of the equation $z^{d}=f$.

The solution of the lifting problem in this simple example is completely understood. We shall see that the general solution is based on this prototypical case. Interestingly, it is also the case with the worst loss of regularity.

The following theorem is a consequence of a result of Ghisi and Gobbino [6].

Theorem 3.1. Let $d$ be a positive integer and let $I \subseteq \mathbb{R}$ be an open bounded interval. Assume that $f: I \rightarrow \mathbb{C}$ is a continuous function such that $f^{d}=g \in C^{d-1,1}(\bar{I})$. Then we have $f^{\prime} \in L_{w}^{d^{\prime}}(I)$ and

$$
\left\|f^{\prime}\right\|_{d^{\prime}, w, I} \leq C(d) \max \left\{\left(\operatorname{Lip}_{I}\left(g^{(d-1)}\right)\right)^{1 / d}|I|^{1 / d^{\prime}},\left\|g^{\prime}\right\|_{L^{\infty}(I)}^{1 / d}\right\}
$$

In other words any continuous lift $f$ over $\sigma(z)=z^{d}$ of a curve in $C^{d-1,1}(\bar{I}, \sigma(\mathbb{C}))=C^{d-1,1}(\bar{I})$ is absolutely continuous and $f^{\prime} \in L_{w}^{d^{\prime}}(I)$ with the uniform bound (3.1).

Remark 3.2. This result is optimal: in general, $f^{\prime}$ is not in $L^{d^{\prime}}$ even if $g$ is real analytic (consider $g(t)=t$ ). On the other hand, if $g$ is only of class $C^{d-1, \beta}(\bar{I})$ for every $\beta<1$, then $f$ does in general not need to have bounded variation in $I$ (see [6, Example 4.4]).

Remark 3.3. If we consider the real representation of $C_{d}$ on $\mathbb{R}^{2}$ by rotation, basic invariants are given by

$$
\sigma_{1}(x, y)=z \bar{z}, \quad \sigma_{2}(x, y)=\operatorname{Re}\left(z^{d}\right), \quad \sigma_{3}(x, y)=\operatorname{Im}\left(z^{d}\right), \quad \text { where } \quad z=x+\mathrm{i} y,
$$

with the relation $\sigma_{1}^{d}=\sigma_{2}^{2}+\sigma_{3}^{2}$. Let $f$ be a map that takes values in $\sigma\left(\mathbb{R}^{2}\right)$, where $\sigma=\left(\sigma_{1}, \sigma_{2}, \sigma_{3}\right)$, and which is smooth as a map into $\mathbb{R}^{3}$. Then the constraints $f$ has to fulfill, in contrast to the complex case where there are no constrains, give reasons for the more regular lifting in the real case (cf. Theorem 1.9).

For instance, suppose that $f$ is a smooth complex valued function. By Theorem 1.9 and the previous paragraph, the equation $z^{d}=f$ has a solution of class $W^{1, \infty}$ provided that $|f|^{2 / d}$ is of class $C^{d-1,1}$. Observe that for $d=2$ and $f \geq 0$ this condition is automatically fulfilled; it corresponds to the hyperbolic case. 


\section{Reduction to slice representations}

Let $G \circlearrowleft V$ be a complex finite-dimensional representation of a finite group $G$. Suppose that $\sigma=\left(\sigma_{1}, \ldots, \sigma_{n}\right)$ is a system of homogeneous basic invariants. Let $V^{G}=\{v \in V: G v=v\}$ be the linear subspace of invariant vectors. It is the subspace of all vectors $v$ for which the isotropy subgroup $G_{v}=\{g \in G: g v=v\}$ is equal to $G$.

\subsection{Removing invariant vectors}

Since finite groups are linearly reductive, there exists a unique subrepresentation $V^{\prime} \subseteq V$ such that $V=V^{G} \oplus V^{\prime}$ (cf. [5, Theorem 2.2.5]). Then $\mathbb{C}[V]^{G}=\mathbb{C}\left[V^{G}\right] \otimes \mathbb{C}\left[V^{\prime}\right]^{G}$ and $V / G=$ $V^{G} \times V^{\prime} / G$. A system of basic invariants of $\mathbb{C}[V]^{G}$ is given by a system of linear coordinates on $V^{G}$ together with a system of basic invariants of $\mathbb{C}\left[V^{\prime}\right]^{G}$. Hence the following lemma is immediate.

Lemma 4.1. Any lift $\bar{f}$ of a mapping $f=\left(f_{0}, f_{1}\right)$ in $V^{G} \times V^{\prime} / G$ has the form $\bar{f}=\left(f_{0}, \bar{f}_{1}\right)$, where $\bar{f}_{1}$ is a lift of $f_{1}$.

Consequently, we may assume without loss of generality that $V^{G}=\{0\}$.

\subsection{Luna's slice theorem}

Let us recall Luna's slice theorem. Here we just assume that $V$ is a rational representation of a linearly reductive group $G$. The categorical quotient $\pi: V \rightarrow V / / G$ is the affine variety with the coordinate ring $\mathbb{C}[V]^{G}$ together with the projection $\pi$ induced by the inclusion $\mathbb{C}[V]^{G} \hookrightarrow \mathbb{C}[V]$. In this setting $\pi$ does not separate orbits, but for each element $z \in V / / G$ there is a unique closed orbit in the fiber $\pi^{-1}(z)$. If $G v$ is a closed orbit, then $G_{v}$ is again linearly reductive. We say that $U \subseteq V$ is $G$-saturated if $\pi^{-1}(\pi(U))=U$.

Theorem 4.2 ([10], [18, Theorem 5.3]). Let $G v$ be a closed orbit. Choose a $G_{v^{-}}$splitting $T_{v}(G v) \oplus N_{v}$ of $V \cong T_{v} V$ and let $\varphi$ denote the mapping

$$
G \times_{G_{v}} N_{v} \rightarrow V, \quad[g, n] \mapsto g(v+n) .
$$

There is an affine open $G$-saturated subset $U$ of $V$ and an affine open $G_{v}$-saturated neighborhood $B_{v}$ of 0 in $N_{v}$ such that

$$
\varphi: G \times_{G_{v}} B_{v} \rightarrow U
$$

and the induced mapping

$$
\bar{\varphi}:\left(G \times_{G_{v}} B_{v}\right) / / G \rightarrow U / / G
$$

are étale. Moreover, $\varphi$ and the natural mapping $G \times_{G_{v}} B_{v} \rightarrow B_{v} / / G_{v}$ induce a $G$-isomorphism of $G \times_{G_{v}} B_{v}$ with $U \times_{U / / G}\left(B_{v} / / G_{v}\right)$.

Corollary 4.3 ([10], [18, Corollary 5.4]). In the setting of Theorem 4.2, $G_{y}$ is conjugate to a subgroup of $G_{v}$ for all $y \in U$. Choose a $G$-saturated neighborhood $\bar{B}_{v}$ of 0 in $B_{v}$ (classical topology) such that the canonical mapping $\bar{B}_{v} / / G_{v} \rightarrow \bar{U} / / G$ is a complex analytic isomorphism, where $\bar{U}=\pi^{-1}\left(\bar{\varphi}\left(\left(G \times_{G_{v}} B_{v}\right) / / G\right)\right)$. Then $\bar{U}$ is a $G$-saturated neighborhood of $v$ and $\varphi: G \times_{G_{v}}$ $\bar{B}_{v} \rightarrow \bar{U}$ is biholomorphic. 


\subsection{Uniform slice reduction}

Let $\left\{\tau_{i}\right\}_{i=1}^{m}$ be a system of generators of $\mathbb{C}\left[N_{v}\right]^{G_{v}}$ and let $\tau=\left(\tau_{1}, \ldots, \tau_{m}\right): N_{v} \rightarrow \mathbb{C}^{m}$ be the associated mapping. Consider the slice

$$
S_{v}:=v+\bar{B}_{v}
$$

where $\bar{B}_{v}$ is the neighborhood from Corollary 4.3 .

Lemma 4.4. Let $a=\left(a_{1}, \ldots, a_{n}\right)$ be a curve in $\sigma(V)$ with $a_{k} \neq 0$ and such that the curve

$$
\underline{a}:=\left(a_{k}{ }^{-d_{1} / d_{k}} a_{1}, \ldots, a_{k}{ }^{-d_{n} / d_{k}} a_{n}\right)
$$

lies in $\sigma\left(U_{v}\right)$, where $U_{v}$ is a neighborhood of $v$ in $S_{v}$. Composition of the curve $\underline{a}-\sigma(v)$ with the analytic isomorphism of Corollary 4.3 gives a curve $\underline{b}=\left(\underline{b}_{1}, \ldots, \underline{b}_{m}\right)$ in $\tau\left(U_{v}-v\right)$ and

$$
b=\left(b_{1}, \ldots, b_{m}\right):=\left(a_{k}^{e_{1} / d_{k}} \underline{b}_{1}, \ldots, a_{k}^{e_{m} / d_{k}} \underline{b}_{m}\right), \quad e_{i}=\operatorname{deg} \tau_{i},
$$

is a curve in $\tau\left(N_{v}\right)$. If $\bar{b}$ is a lift of $b$ over $\tau$ then

$$
a_{k}^{1 / d_{k}} v+\bar{b}
$$

is a lift of a over $\sigma$.

Proof. The curve $a_{k}^{-1 / d_{k}} \bar{b}$ is a lift of $\underline{b}$ over $\tau$, indeed by homogeneity,

$$
\tau_{i}\left(a_{k}^{-1 / d_{k}} \bar{b}\right)=a_{k}^{-e_{i} / d_{k}} \tau_{i}(\bar{b})=a_{k}^{-e_{i} / d_{k}} b_{i}=\underline{b}_{i} .
$$

Thus $a_{k}{ }^{-1 / d_{k}} \bar{b}+v$ is a lift of $\underline{a}$ over $\sigma$. By homogeneity, we find $\sigma_{i}\left(\bar{b}+a_{k}{ }^{1 / d_{k}} v\right)=a_{k}{ }^{d_{i} / d_{k}} \underline{a}_{i}=a_{i}$ as required.

The following lemma shows that the maximal degree of the basic invariants does not increase by passing to a slice representation. It can be shown in analogy to [8, Lemma 2.4] or [14].

Lemma 4.5. Assume that the systems of basic invariants $\left\{\sigma_{j}\right\}_{j=1}^{n}$ and $\left\{\tau_{i}\right\}_{i=1}^{m}$ are minimal and set $e:=\max _{i} e_{i}=\max _{i} \operatorname{deg} \tau_{i}$. Then $e \leq d$.

In order to make the slice reduction uniform, we consider the set

$$
K:=\left(\bigcup_{k=1}^{n}\left\{\left(\underline{a}_{1}, \ldots, \underline{a}_{n}\right) \in \mathbb{C}^{n}: \underline{a}_{k}=1,\left|\underline{a}_{j}\right| \leq 1 \text { for } j \neq k\right\}\right) \cap \sigma(V),
$$

which is compact, since $\sigma(V)$ is closed. For each point $\underline{p} \in K$ choose $v \in \sigma^{-1}(\underline{p})$. Then the collection $\left\{\sigma\left(U_{v}\right)\right\}$ for all such $v$ is a cover of $K$ by sets $\sigma\left(\bar{U}_{v}\right)$ that are open in the trace topology on $\sigma(V)$ and on which the conclusion of Lemma 4.4 holds. Choose a finite subcover

$$
\mathcal{B}:=\left\{B_{\delta}\right\}_{\delta \in \Delta}=\left\{\sigma\left(U_{v_{\delta}}\right)\right\}_{\delta \in \Delta} .
$$

Then there exists $\rho>0$ such that for every $\underline{p} \in K$ there is a $\delta \in \Delta$ such that

$$
B_{\rho}(\underline{p}) \cap \sigma(V) \subseteq B_{\delta},
$$

where $B_{\rho}(\underline{p})$ is the open ball with radius $\rho$ centered at $\underline{p}$.

Definition 4.6. We refer to this data as the uniform slice reduction of the representation $G \circlearrowleft V$, in particular, we call $\rho>0$ from (4.2) the uniform reduction radius. 


\section{Estimates for a curve in $\sigma(V)$}

In the next three sections we discuss preparatory lemmas for the proof of Theorem 1.1 which is then given in Section 8 .

\subsection{An interpolation inequality}

For an interval $I \subseteq \mathbb{R}$ and a function $f: I \rightarrow \mathbb{C}$ we set

$$
V_{I}(f):=\sup _{t, s \in I}|f(t)-f(s)|=\operatorname{diam} f(I) .
$$

Lemma 5.1 ([16, Lemma 4]). Let $I \subseteq \mathbb{R}$ be a bounded open interval, $m \in \mathbb{N}_{>0}$, and $\alpha \in(0,1]$. If $f \in C^{m, \alpha}(\bar{I})$, then for all $t \in I$ and $s=1, \ldots, m$,

$$
\left|f^{(s)}(t)\right| \leq C|I|^{-s}\left(V_{I}(f)+V_{I}(f)^{(m+\alpha-s) /(m+\alpha)}\left(\operatorname{Höld}_{\alpha, I}\left(f^{(m)}\right)\right)^{s /(m+\alpha)}|I|^{s}\right),
$$

for a universal constant $C$ depending only on $m$ and $\alpha$.

\subsection{The local setup}

Let $G \circlearrowleft V$ be a complex finite-dimensional representation of a finite group $G$. Assume $V^{G}=\{0\}$. Let $\sigma=\left(\sigma_{1}, \ldots, \sigma_{n}\right)$ be a system of homogeneous basic invariants of degrees $d_{1}, \ldots, d_{n}$ and let $d:=\max _{j} d_{j}$. Let $a \in C^{d-1,1}(\bar{I}, \sigma(V))$, where $I \subseteq \mathbb{R}$ is a bounded open interval.

It will be crucial to consider the radicals $a_{j}^{1 / d_{j}}$ of the components $a_{j}$ of $a$ which is justified by the following remark.

Remark 5.2. Every continuous selection $f$ of the multi-valued function $a_{j}^{1 / d_{j}}$ is absolutely continuous on $I$, by Theorem 3.1. (Clearly, continuous selections exist in this case.) Moreover, $\left\|f^{\prime}\right\|_{L^{1}(I)}$ is independent of the choice of the selection. Indeed, if $g$ is a different continuous selection then on each connected component $J$ of $I \backslash\left\{t: a_{j}(t)=0\right\}$ the functions $f$ and $g$ just differ by multiplication with a fixed $d_{j^{-}}$th root of unity. Thus $\left\|f^{\prime}\right\|_{L^{1}(J)}=\left\|g^{\prime}\right\|_{L^{1}(J)}$. The $\mathbb{C}$ valued version of Lemma 2.1 implies that $\left\|f^{\prime}\right\|_{L^{1}(I)}=\left\|g^{\prime}\right\|_{L^{1}(I)}$.

Henceforth we fix one continuous selection of $a_{j}^{1 / d_{j}}$ and denote it by

$$
\hat{a}_{j}: I \rightarrow \mathbb{C}
$$

as well as, abusing notation, by $a_{j}^{1 / d_{j}}$. We will also consider the absolutely continuous curve

$$
\hat{a}=\left(\hat{a}_{1}, \ldots, \hat{a}_{n}\right): I \rightarrow \mathbb{C}^{n} .
$$

Suppose that $t_{0} \in I$ and $k \in\{1, \ldots, n\}$ are such that

$$
\left|\hat{a}_{k}\left(t_{0}\right)\right|=\max _{1 \leq j \leq n}\left|\hat{a}_{j}\left(t_{0}\right)\right| \neq 0 .
$$

Assume further that, for some fixed positive constant $B<1 / 3$,

$$
\left\|\hat{a}^{\prime}\right\|_{L^{1}(I)} \leq B\left|\hat{a}_{k}\left(t_{0}\right)\right| .
$$

At this point we just demand that the constant $B$ is fixed and smaller than $1 / 3$; in the proof of Theorem 1.1 we will additionally specify $B$ depending on the uniform reduction radius $\rho>0$ and the maximal degree $d$, see (8.1), and it is going to be fixed along said proof. In accordance with (2.2), $\left\|\hat{a}^{\prime}\right\|_{L^{1}(I)}=\sum_{j=1}^{n}\left\|\hat{a}_{j}^{\prime}\right\|_{L^{1}(I)}$.

Definition 5.3. By admissible data for $G \circlearrowleft V$ me mean a tuple $\left(a, I, t_{0}, k\right)$, where $a \in$ $C^{d-1,1}(\bar{I}, \sigma(V))$ is a curve in $\sigma(V)$ for a representation $G \circlearrowleft V$ with $V^{G}=\{0\}$ defined on an open bounded interval $I$ such that $t_{0} \in I$ and $k \in\{1, \ldots, n\}$ satisfy (5.1) and (5.2). 


\subsection{The reduced curve $\underline{a}$}

Let $\left(a, I, t_{0}, k\right)$ be admissible data for $G \circlearrowleft V$. We shall see in the next lemma that $a_{k}$ does not vanish on the interval $I$ and so the curve

$$
\begin{aligned}
\underline{a}: \quad I & \rightarrow\left\{\left(\underline{a}_{1}, \ldots, \underline{a}_{n}\right) \in \mathbb{C}^{n}: \underline{a}_{k}=1\right\}, \\
& t \mapsto \underline{a}(t):=\left(a_{k}^{-d_{1} / d_{k}} a_{1}, \ldots, a_{k}^{-d_{n} / d_{k}} a_{n}\right)(t)=\left(\left(\hat{a}_{k}^{-1} \hat{a}_{1}\right)^{d_{1}}, \ldots,\left(\hat{a}_{k}^{-1} \hat{a}_{n}\right)^{d_{n}}\right)(t)
\end{aligned}
$$

is well-defined. The homogeneity of the basic invariants implies that $\underline{a}(I) \subseteq \sigma(V)$.

Lemma 5.4. Let $\left(a, I, t_{0}, k\right)$ be admissible data for $G \circlearrowleft V$. Then for all $t \in I$ and $j=1, \ldots, n$,

$$
\begin{aligned}
& \left|\hat{a}_{j}(t)-\hat{a}_{j}\left(t_{0}\right)\right| \leq B\left|\hat{a}_{k}\left(t_{0}\right)\right|, \\
& \frac{2}{3}<1-B \leq\left|\frac{\hat{a}_{k}(t)}{\hat{a}_{k}\left(t_{0}\right)}\right| \leq 1+B<\frac{4}{3}, \\
& \left|\hat{a}_{j}(t)\right| \leq \frac{4}{3}\left|\hat{a}_{k}\left(t_{0}\right)\right| \leq 2\left|\hat{a}_{k}(t)\right| .
\end{aligned}
$$

The length of the curve $\underline{a}$ is bounded by $3 d^{2} 2^{d} B$.

Proof. First (5.4) is a consequence of (5.2),

$$
\left|\hat{a}_{j}(t)-\hat{a}_{j}\left(t_{0}\right)\right|=\left|\int_{t_{0}}^{t} \hat{a}_{j}^{\prime} \mathrm{d} s\right| \leq\left\|\hat{a}_{j}^{\prime}\right\|_{L^{1}(I)} \leq B\left|\hat{a}_{k}\left(t_{0}\right)\right| .
$$

Setting $j=k$ in (5.4) easily implies (5.5). Together with (5.1), the inequalities (5.4) and (5.5) give (5.6). In order to estimate the length of $\underline{a}$ observe that

$$
\underline{a}_{j}^{\prime}=\partial_{t}\left(\left(\hat{a}_{k}^{-1} \hat{a}_{j}\right)^{d_{j}}\right)=d_{j}\left(\hat{a}_{k}^{-1} \hat{a}_{j}\right)^{d_{j}-1}\left(\hat{a}_{k}^{-1} \hat{a}_{j}^{\prime}-\hat{a}_{k}^{-2} \hat{a}_{j} \hat{a}_{k}^{\prime}\right) .
$$

Since $\left|\hat{a}_{k}^{-1} \hat{a}_{j}\right| \leq 2$, by (5.6), and thanks to (5.5) we obtain

$$
\left|\underline{a}_{j}^{\prime}\right| \leq 3 d 2^{d}\left|\hat{a}_{k}\left(t_{0}\right)\right|^{-1}\left(\left|\hat{a}_{j}^{\prime}\right|+\left|\hat{a}_{k}^{\prime}\right|\right) \text {. }
$$

Consequently, using (5.2),

$$
\int_{I}\left|\underline{a}^{\prime}\right| \mathrm{d} s \leq 3 d^{2} 2^{d} B
$$

as required.

\section{The estimates after reduction to a slice representation}

\subsection{The reduced local setup}

Let $\left(a, I, t_{0}, k\right)$ be admissible data for $G \circlearrowleft V$ such that for all $j=1, \ldots, n$ and $s=1, \ldots, d-1$,

$$
\begin{aligned}
& \left\|a_{j}^{(s)}\right\|_{L^{\infty}(I)} \leq C(d)|I|^{-s}\left|\hat{a}_{k}\left(t_{0}\right)\right|^{d_{j}}, \\
& \operatorname{Lip}_{I}\left(a_{j}^{(d-1)}\right) \leq C(d)|I|^{-d}\left|\hat{a}_{k}\left(t_{0}\right)\right|^{d_{j}} .
\end{aligned}
$$

Here $C(d)$ is a positive constant which depends only on $d$; in Lemma 8.1 we will see that the assumptions of Theorem 1.1 imply (6.1) on suitable intervals $I$, and the proof of Lemma 8.1 will provide a specific value for $C(d)$. 
Additionally, we suppose that the curve $\underline{a}$ (defined in (5.3)) lies entirely in one of the balls $B_{\rho}(\underline{p})$ from (4.2). By Lemma 4.4, we obtain a curve $b \in C^{d-1,1}(\bar{I}, \tau(W))$, where $H \circlearrowleft W$ with $H=G_{v}$ and $W=N_{v}$ is a slice representation of $G \circlearrowleft V$ and

$$
b_{i}=a_{k}^{e_{i} / d_{k}} \psi_{i}\left(a_{k}^{-d_{1} / d_{k}} a_{1}, \ldots, a_{k}^{-d_{n} / d_{k}} a_{n}\right), \quad i=1, \ldots, m,
$$

where $e_{i}=\operatorname{deg} \tau_{i}$ and the $\psi_{i}$ are analytic functions which are bounded on their domain together with all their partial derivatives (this may be achieved by slightly shrinking the domain).

In accordance with Remark 5.2 we denote by

$$
\hat{b}_{i}: I \rightarrow \mathbb{C}
$$

a fixed continuous selection of $b_{i}^{1 / e_{i}}$. Sometimes it will also be convenient to use just the symbol $b_{i}^{1 / e_{i}}$ for $\hat{b}_{i}$. We set

$$
\hat{b}=\left(\hat{b}_{1}, \ldots, \hat{b}_{m}\right): I \rightarrow \mathbb{C}^{m} .
$$

Hence (6.2) can also be written as

$$
b_{i}=\hat{a}_{k}^{e_{i}} \psi_{i}\left(\hat{a}_{k}^{-d_{1}} a_{1}, \ldots, \hat{a}_{k}^{-d_{n}} a_{n}\right)=\hat{a}_{k}^{e_{i}} \cdot \psi_{i} \circ \underline{a} .
$$

Thanks to Lemma 4.1 we may assume that $W^{H}=\{0\}$.

Definition 6.1. By reduced admissible data for $G \circlearrowleft V$ me mean a tuple $\left(a, I, t_{0}, k ; b\right)$, where $\left(a, I, t_{0}, k\right)$ is admissible data for $G \circlearrowleft V$ satisfying (6.1) such that $\underline{a}$ lies entirely in one of the balls $B_{\rho}(\underline{p})$ from (4.2) and $b \in C^{d-1,1}(\bar{I}, \tau(W))$ is a curve resulting from Lemma 4.4 and thus satisfies (6.2).

The goal of this section is to show that the bounds (6.1) are inherited by the curve $b$ on suitable subintervals. This requires some preparation.

\subsection{Pointwise estimates for the derivatives of $b$ on $I$}

Lemma 6.2. Let $\left(a, I, t_{0}, k ; b\right)$ be reduced admissible data for $G \circlearrowleft V$. Then for all $i=1, \ldots, m$ and $s=1, \ldots, d-1$,

$$
\begin{aligned}
& \left\|b_{i}^{(s)}\right\|_{L^{\infty}(I)} \leq C|I|^{-s}\left|\hat{a}_{k}\left(t_{0}\right)\right|^{e_{i}}, \\
& \operatorname{Lip}_{I}\left(b_{i}^{(d-1)}\right) \leq C|I|^{-d}\left|\hat{a}_{k}\left(t_{0}\right)\right|^{e_{i}},
\end{aligned}
$$

where $C$ is a constant depending only on $d$ and on the functions $\psi_{i}$.

Proof. Let us prove the first estimate in (6.3). Let $F$ be any $C^{d}$-function defined on an open set $U \subseteq \mathbb{C}^{n}$ that contains $\underline{a}(I)$ and assume $\|F\|_{C^{d}(\bar{U})}<\infty$. We claim that, for $s=1, \ldots, d-1$,

$$
\left\|\partial_{t}^{s}(F \circ \underline{a})\right\|_{L^{\infty}(I)} \leq C|I|^{-s}
$$

where $C$ is a constant depending only on $d$ and $\|F\|_{C^{d}(\bar{U})}$. For any real exponent $r$, Faà di Bruno's formula implies

$$
\partial_{t}^{s}\left(a_{j}^{r}\right)=\sum_{\ell \geq 1}^{s} \sum_{\gamma \in \Gamma(\ell, s)} c_{\gamma, \ell, r} a_{j}^{r-\ell} a_{j}^{\left(\gamma_{1}\right)} \cdots a_{j}^{\left(\gamma_{\ell}\right)},
$$


where $\Gamma(\ell, s)=\left\{\gamma \in \mathbb{N}_{>0}^{\ell}:|\gamma|=s\right\}$ and

$$
c_{\gamma, \ell, r}=\frac{s !}{\ell ! \gamma !} r(r-1) \cdots(r-\ell+1) .
$$

By (6.1) and (5.5), this implies for $j=k$

$$
\begin{aligned}
\left\|\partial_{t}^{s}\left(a_{k}^{r}\right)\right\|_{L^{\infty}(I)} & \leq \sum_{\ell \geq 1}^{s} \sum_{\gamma \in \Gamma(\ell, s)} c_{\gamma, \ell, r}\left\|a_{k}^{r-\ell}\right\|_{L^{\infty}(I)}\left\|a_{k}^{\left(\gamma_{1}\right)}\right\|_{L^{\infty}(I)} \cdots\left\|a_{k}^{\left(\gamma_{\ell}\right)}\right\|_{L^{\infty}(I)} \\
& \leq C(d) \sum_{\ell \geq 1}^{s} \sum_{\gamma \in \Gamma(\ell, s)} c_{\gamma, \ell, r}\left|a_{k}\left(t_{0}\right)\right|^{r-\ell}|I|^{-s}\left|a_{k}\left(t_{0}\right)\right|^{\ell} \\
& \leq C(d)|I|^{-s}\left|a_{k}\left(t_{0}\right)\right|^{r} .
\end{aligned}
$$

Together with the Leibniz formula,

$$
\partial_{t}^{s}\left(a_{k}^{-d_{j} / d_{k}} a_{j}\right)=\sum_{q=0}^{s}\left(\begin{array}{l}
s \\
q
\end{array}\right) a_{j}^{(q)} \partial_{t}^{s-q}\left(a_{k}^{-d_{j} / d_{k}}\right),
$$

(6.6) and (6.1) lead to

$$
\left\|\partial_{t}^{s}\left(a_{k}^{-d_{j} / d_{k}} a_{j}\right)\right\|_{L^{\infty}(I)} \leq C(d)|I|^{-s} .
$$

Again by the Leibniz formula,

$$
\begin{aligned}
\partial_{t}(F \circ \underline{a}) & =\sum_{j=1}^{n}\left(\left(\partial_{j} F\right) \circ \underline{a}\right) \partial_{t}\left(a_{k}^{-d_{j} / d_{k}} a_{j}\right), \\
\partial_{t}^{s}(F \circ \underline{a}) & =\sum_{j=1}^{n} \partial_{t}^{s-1}\left(\left(\left(\partial_{j} F\right) \circ \underline{a}\right) \partial_{t}\left(a_{k}^{-d_{j} / d_{k}} a_{j}\right)\right) \\
& =\sum_{j=1}^{n} \sum_{p=0}^{s-1}\left(\begin{array}{c}
s-1 \\
p
\end{array}\right) \partial_{t}^{p}\left(\left(\partial_{j} F\right) \circ \underline{a}\right) \partial_{t}^{s-p}\left(a_{k}^{-d_{j} / d_{k}} a_{j}\right) .
\end{aligned}
$$

For $s=1$ we immediately get (6.4). For $1<s \leq d-1$, we may argue by induction on $s$. By induction hypothesis,

$$
\left\|\partial_{t}^{p}\left(\left(\partial_{j} F\right) \circ \underline{a}\right)\right\|_{L^{\infty}(I)} \leq C\left(d,\left\|\partial_{j} F\right\|_{C^{s}(\bar{U})}\right)|I|^{-p},
$$

for $p=1, \ldots, s-1$. Together with (6.7) this entails (6.4).

Now the first part of (6.3) is a consequence of (6.2), (6.6) (for $r=e_{i} / d_{k}$ ), and (6.4) (applied to $F=\psi_{i}$ ).

For the second part of (6.3) observe that for functions $f_{1}, \ldots, f_{m}$ on $I$ we have

$$
\operatorname{Lip}_{I}\left(f_{1} f_{2} \cdots f_{m}\right) \leq \sum_{i=1}^{m} \operatorname{Lip}_{I}\left(f_{i}\right)\left\|f_{1}\right\|_{L^{\infty}(I)} \cdots\left\|\widehat{f_{i} \|_{L^{\infty}(I)}} \cdots\right\| f_{m} \|_{L^{\infty}(I)} .
$$

Applying it to (6.5) and using

$$
\operatorname{Lip}_{I}\left(a_{j}^{r-\ell}\right) \leq|r-\ell|\left\|a_{j}^{r-\ell-1}\right\|_{L^{\infty}(I)}\left\|a_{j}^{\prime}\right\|_{L^{\infty}(I)}
$$

we find, as in the derivation of (6.6),

$$
\operatorname{Lip}_{I}\left(\partial_{t}^{d-1}\left(a_{k}^{r}\right)\right) \leq C(d, r)|I|^{-d}\left|a_{k}\left(t_{0}\right)\right|^{r} .
$$


As above this leads to

$$
\operatorname{Lip}_{I}\left(\partial_{t}^{d-1}\left(a_{k}^{-d_{j} / d_{k}} a_{j}\right)\right) \leq C(d)|I|^{-d},
$$

and

$$
\operatorname{Lip}_{I}\left(\partial_{t}^{d-1}(F \circ \underline{a})\right) \leq C\left(d,\|F\|_{C^{d}(\bar{U})}\right)|I|^{-d},
$$

and finally to the second part of (6.3).

\subsection{Integral bounds for $\hat{b}^{\prime}$}

Recall that $e=\max _{i} e_{i}=\max _{i} \operatorname{deg} \tau_{i}$ and $e^{\prime}=e /(e-1)$.

Corollary 6.3. Let $\left(a, I, t_{0}, k ; b\right)$ be reduced admissible data for $G \circlearrowleft V$. Then, for all $1 \leq p<e^{\prime}$ and all $i=1, \ldots, m$,

$$
\left\|\hat{b}_{i}^{\prime}\right\|_{L^{p}(I)}^{*} \leq C|I|^{-1}\left|\hat{a}_{k}\left(t_{0}\right)\right|
$$

for a constant $C$ which depends only on $d, p$, and the constant in (6.3).

Proof. Notice that, by Lemma 4.5, we have $e \leq d$. By (3.1) and (6.3),

$$
\begin{aligned}
\left\|\hat{b}_{i}^{\prime}\right\|_{e_{i}^{\prime}, w, I} & =\left\|\left(b_{i}^{1 / e_{i}}\right)^{\prime}\right\|_{e_{i}^{\prime}, w, I} \leq C\left(e_{i}\right) \max \left\{\left(\operatorname{Lip}_{I}\left(b_{i}^{\left(e_{i}-1\right)}\right)\right)^{1 / e_{i}}|I|^{1 / e_{i}^{\prime}},\left\|b_{i}^{\prime}\right\|_{L^{\infty}(I)}^{1 / e_{i}}\right\} \\
& \leq C|I|^{-1+1 / e_{i}^{\prime}}\left|\hat{a}_{k}\left(t_{0}\right)\right|,
\end{aligned}
$$

or equivalently,

$$
\left\|\hat{b}_{i}^{\prime}\right\|_{e_{i}^{\prime}, w, I}^{*} \leq C|I|^{-1}\left|\hat{a}_{k}\left(t_{0}\right)\right| .
$$

This entails (6.8) in view of (2.1).

\subsection{Special subintervals of $I$ and estimates on them}

Let $\left(a, I, t_{0}, k ; b\right)$ be reduced admissible data for $G \circlearrowleft V$.

Suppose that $t_{1} \in I$ and $\ell \in\{1, \ldots, m\}$ are such that

$$
\left|\hat{b}_{\ell}\left(t_{1}\right)\right|=\max _{1 \leq i \leq m}\left|\hat{b}_{i}\left(t_{1}\right)\right| \neq 0
$$

By (5.6) and (6.2), for all $t \in I$ and $i=1, \ldots, m$,

$$
\left|\hat{b}_{i}(t)\right| \leq C_{1}\left|\hat{a}_{k}\left(t_{0}\right)\right|
$$

where the constant $C_{1}$ depends only on the functions $\psi_{i}$. Thanks to (6.10) we can choose a constant $D<1 / 3$ and an open interval $J$ with $t_{1} \in J \subseteq I$ such that

$$
|J||I|^{-1}\left|\hat{a}_{k}\left(t_{0}\right)\right|+\left\|\hat{b}^{\prime}\right\|_{L^{1}(J)}=D\left|\hat{b}_{\ell}\left(t_{1}\right)\right|,
$$

where $\left\|\hat{b}^{\prime}\right\|_{L^{1}(J)}=\sum_{i=1}^{m}\left\|\hat{b}_{i}^{\prime}\right\|_{L^{1}(J)}$. It suffices to take $D<C_{1}^{-1}$, where $C_{1}$ is the constant in (6.10). Here we use that $\hat{b}_{i}$ is absolutely continuous, by Theorem 3.1 .

We will now see that on the interval $J$ the estimates of Section 5 hold for $b_{i}$ instead of $a_{j}$. 
Lemma 6.4. Let $\left(a, I, t_{0}, k ; b\right)$ be reduced admissible data for $G \circlearrowleft V$. Assume that $t_{1} \in I$ and $\ell \in\{1, \ldots, m\}$ are such that (6.9) holds and let $D$ and $J$ be as in (6.11). Then, for all $t \in J$ and $i=1, \ldots, m$,

$$
\begin{aligned}
& \left|\hat{b}_{i}(t)-\hat{b}_{i}\left(t_{1}\right)\right| \leq D\left|\hat{b}_{\ell}\left(t_{1}\right)\right|, \\
& \frac{2}{3}<1-D \leq\left|\frac{\hat{b}_{\ell}(t)}{\hat{b}_{\ell}\left(t_{1}\right)}\right| \leq 1+D<\frac{4}{3}, \\
& \left|\hat{b}_{i}(t)\right| \leq \frac{4}{3}\left|\hat{b}_{\ell}\left(t_{1}\right)\right| \leq 2\left|\hat{b}_{\ell}(t)\right| .
\end{aligned}
$$

The length of the curve

$$
J \ni t \mapsto \underline{b}(t):=\left(b_{\ell}^{-e_{1} / e_{\ell}} b_{1}, \ldots, b_{\ell}^{-e_{m} / e_{\ell}} b_{m}\right)(t)=\left(\left(\hat{b}_{\ell}^{-1} \hat{b}_{1}\right)^{e_{1}}, \ldots,\left(\hat{b}_{\ell}^{-1} \hat{b}_{m}\right)^{e_{m}}\right)(t)
$$

in $\tau(W)$ is bounded by $3 e^{2} 2^{e} D$. For all $i=1, \ldots, m$ and $s=1, \ldots, d-1$,

$$
\begin{aligned}
& \left\|b_{i}^{(s)}\right\|_{L^{\infty}(J)} \leq C|J|^{-s}\left|\hat{b}_{\ell}\left(t_{1}\right)\right|^{e_{i}}, \\
& \operatorname{Lip}_{J}\left(b_{i}^{(d-1)}\right) \leq C|J|^{-d}\left|\hat{b}_{\ell}\left(t_{1}\right)\right|^{e_{i}},
\end{aligned}
$$

for a universal constant $C$ depending only on $d$ and $\psi_{i}$.

Proof. The proof of (6.12)-(6.14) is analogous to the proof of Lemma 5.4; use (6.9) and (6.11) instead of (5.1) and (5.2). The bound for the length of the curve $J \ni t \mapsto \underline{b}(t)$ (which is well-defined by (6.13)) follows from (6.11)-(6.14); see the proof of Lemma 5.4.

Let us prove (6.15). By (6.3), for $i=1, \ldots, m$ and $s=1, \ldots, d-1$

$$
\begin{aligned}
& \left\|b_{i}^{(s)}\right\|_{L^{\infty}(I)} \leq C|I|^{-s}\left|\hat{a}_{k}\left(t_{0}\right)\right|^{e_{i}}, \\
& \operatorname{Lip}_{I}\left(b_{i}^{(d-1)}\right) \leq C|I|^{-d}\left|\hat{a}_{k}\left(t_{0}\right)\right|^{e_{i}},
\end{aligned}
$$

where $C=C\left(d, \psi_{i}\right)$. Recall that $e \leq d$.

For $s \geq e_{i}$ (including the case $s=d$ ), we have $\left(|J||I|^{-1}\right)^{s} \leq\left(|J||I|^{-1}\right)^{e_{i}}$ and thus

$$
|I|^{-s}\left|\hat{a}_{k}\left(t_{0}\right)\right|^{e_{i}} \leq|J|^{-s}\left(|J||I|^{-1}\left|\hat{a}_{k}\left(t_{0}\right)\right|\right)^{e_{i}} \leq|J|^{-s}\left|\hat{b}_{\ell}\left(t_{1}\right)\right|^{e_{i}},
$$

where the second inequality follows from (6.11). Hence (6.16) implies (6.15).

For $t \in J$ and $s<e_{i}$,

$$
\begin{aligned}
\left|b_{i}^{(s)}(t)\right| & \leq C|J|^{-s}\left(V_{J}\left(b_{i}\right)+V_{J}\left(b_{i}\right)^{\left(e_{i}-s\right) / e_{i}}\left(\operatorname{Lip}_{J}\left(b_{i}^{\left(e_{i}-1\right)}\right)\right)^{s / e_{i}}|J|^{s}\right) & & \text { by Lemma } 5.1 \\
& \leq C_{1}|J|^{-s}\left(\left|\hat{b}_{\ell}\left(t_{1}\right)\right|^{e_{i}}+\left|\hat{b}_{\ell}\left(t_{1}\right)\right|^{e_{i}-s}|J|^{s}|I|^{-s}\left|\hat{a}_{k}\left(t_{0}\right)\right|^{s}\right) & & \text { by }(6.14) \text { and }(6.16) \\
& \leq C_{2}|J|^{-s}\left|\hat{b}_{\ell}\left(t_{1}\right)\right|^{e_{i}}, & & \text { by }(6.11)
\end{aligned}
$$

for constants $C=C\left(e_{i}\right)$ and $C_{h}=C_{h}\left(d, \psi_{i}\right)$.

\section{A special cover by intervals}

In the proof of Theorem 1.1 we shall have to glue local integral bounds on small intervals which result from the splitting process to global bounds. In this section we present a technical result which will allow us to do so.

Let us suppose that $H \circlearrowleft W$ is a complex finite-dimensional representation of a finite group $H$, $\tau=\left(\tau_{1}, \ldots, \tau_{m}\right)$ is a system of homogeneous basic invariants of degree $e_{i}=\operatorname{deg} \tau_{i}$, and $e:=$ $\max _{i} e_{i}$. 


\subsection{Covers by prepared collections of intervals}

Let $I \subseteq \mathbb{R}$ be a bounded open interval and let $b \in C^{e-1,1}(\bar{I}, \tau(W))$. For each point $t_{1}$ in

$$
I^{\prime}:=I \backslash\{t \in I: b(t)=0\}
$$

there exists $\ell \in\{1, \ldots, m\}$ such that (6.9) holds. Assume that there are positive constants $D<1 / 3$ and $L$ such that for all $t_{1} \in I^{\prime}$ there is an open interval $J=J\left(t_{1}\right)$ with $t_{1} \in J \subseteq I$ such that

$$
L|J|+\left\|\hat{b}^{\prime}\right\|_{L^{1}(J)}=D\left|\hat{b}_{\ell}\left(t_{1}\right)\right| .
$$

Note that (6.9) and (7.1) imply (6.13) (cf. the proof of Lemma 6.4); in particular, we have $J \subseteq I^{\prime}$.

This defines a collection $\mathcal{I}:=\left\{J\left(t_{1}\right)\right\}_{t_{1} \in I^{\prime}}$ of open (in the relative topology) intervals which cover $I^{\prime}$. We will prepare this collection in the following way. Let us consider the functions

$$
\begin{array}{ll}
\varphi_{t_{1},+}(s):=L\left(s-t_{1}\right)+\left\|\hat{b}^{\prime}\right\|_{L^{1}\left(\left[t_{1}, s\right)\right)}, & s \geq t_{1}, \\
\varphi_{t_{1},-}(s):=L\left(t_{1}-s\right)+\left\|\hat{b}^{\prime}\right\|_{L^{1}\left(\left(s, t_{1}\right]\right)}, & s \leq t_{1} .
\end{array}
$$

Then $\varphi_{t_{1}, \pm} \geq 0$ are monotonic continuous functions defined for small $\pm\left(s-t_{1}\right) \geq 0$ and satisfying $\varphi_{t_{1}, \pm}\left(t_{1}\right)=0$.

Fix $t_{1} \in I^{\prime}$. Thanks to (7.1) there exist $s_{-}, s_{+} \in \mathbb{R}$ such that

$$
\varphi_{t_{1},-}\left(s_{-}\right)+\varphi_{t_{1},+}\left(s_{+}\right)=D\left|\hat{b}_{\ell}\left(t_{1}\right)\right|
$$

and $J\left(t_{1}\right)=\left(s_{-}, s_{+}\right)$. But there may also be a choice $s_{-}^{\prime}, s_{+}^{\prime} \in \mathbb{R}$ such that this occurs symmetrically, that is

$$
\varphi_{t_{1},-}\left(s_{-}^{\prime}\right)=\varphi_{t_{1},+}\left(s_{+}^{\prime}\right)=\frac{D}{2}\left|\hat{b}_{\ell}\left(t_{1}\right)\right| .
$$

If such a choice $s_{-}^{\prime}, s_{+}^{\prime} \in \mathbb{R}$ exists, we replace $J\left(t_{1}\right)$ in the collection $\mathcal{I}$ by the interval $\left(s_{-}^{\prime}, s_{+}^{\prime}\right)$. (In [16] we said that these are intervals of first kind.) If such a choice does not exist, then we leave $J\left(t_{1}\right)$ in $\mathcal{I}$ unchanged; this happens when we reach the boundary of the interval $I$ before either $\varphi_{t_{1},-}$ or $\varphi_{t_{1},+}$ has grown to the value $(D / 2)\left|\hat{b}_{\ell}\left(t_{1}\right)\right|$. (These intervals were said to be of second kind in [16].)

If a collection $\mathcal{I}$ satisfies this property, we say that it is prepared.

\subsection{A special subcollection of intervals}

Proposition 7.1. Let $I \subseteq \mathbb{R}$ be a bounded open interval. Let $b \in C^{e-1,1}(\bar{I}, \tau(W))$. For each point $t_{1}$ in $I^{\prime}$ fix $\ell \in\{1, \ldots, m\}$ such that (6.9) holds. Let $\mathcal{I}=\left\{J\left(t_{1}\right)\right\}_{t_{1} \in I^{\prime}}$ be a collection of open intervals $J=J\left(t_{1}\right)$ with $t_{1} \in J \subseteq I^{\prime}$ such that:

1. There are positive constants $D<1 / 3$ and $L$ such that for all $t_{1} \in I^{\prime}$ we have (7.1) for $J=J\left(t_{1}\right)$.

2. The collection $\mathcal{I}$ is prepared as explained in Section 7.1.

Then the collection $\mathcal{I}$ has a countable subcollection $\mathcal{J}$ that still covers $I^{\prime}$ and such that every point in $I^{\prime}$ belongs to at most two intervals in $\mathcal{J}$. In particular,

$$
\sum_{J \in \mathcal{J}}|J| \leq 2\left|I^{\prime}\right|
$$

Proof. It follows from the proof of [16, Proposition 2].

Remark 7.2. It is essential for us that $\mathcal{J}$ is a subcollection and not a refinement; by shrinking the intervals we would lose equality in (7.1). We will need this proposition for gluing local $L^{p}$-estimates to global ones. 


\section{Proof of Theorem 1.1}

The proof is based on uniform slice reduction and induction on the order of $G$. We will apply the following convention:

We will no longer explicitly state all the dependencies of the constants. Henceforth, their dependence on the data of the uniform slice reductions will be subsumed by simply indicating that they depend on the representation $G \circlearrowleft V$. This includes the choice of $\sigma$ : different choices of the basic invariants yield different constants. The constants which are uniform in this sense will be denoted by $C=C(G \circlearrowleft V)$ and may vary from line to line.

\section{Outline of the proof}

The proof of Theorem 1.1 is divided into three steps.

Step 1: We check that for any $a \in C^{d-1,1}([\alpha, \beta], \sigma(V))$ and all points $t_{0} \in(\alpha, \beta)$, where $a\left(t_{0}\right) \neq 0$, we can find $k$ and a suitable interval $I$ such that $\left(\left.a\right|_{I}, I, t_{0}, k ; b\right)$, where $b$ is obtained by Lemma 4.4, is reduced admissible data for $G \circlearrowleft V$.

Step 2: The reduced admissible data $\left(\left.a\right|_{I}, I, t_{0}, k ; b\right)$ represents the hypothesis of the inductive argument which is the heart of the proof. It will show that every continuous lift of $b$ is absolutely continuous on $I$ and it will give an $L^{p}$-bound for the first derivative of the lift on $I$.

Step 3: We assemble the proof of Theorem 1.1. The local bounds will be glued to global bounds for lifts of the original curve $a$.

\section{Step 1: The assumptions of Theorem 1.1 imply the local setup of the induction}

Assume that $V^{G}=\{0\}$. Let $a \in C^{d-1,1}([\alpha, \beta], \sigma(V))$. Let $\rho$ be the uniform reduction radius from (4.2). We fix a universal positive constant $B$ satisfying

$$
B<\min \left\{\frac{1}{3}, \frac{\rho}{3 d^{2} 2^{d}}\right\} .
$$

Fix $t_{0} \in(\alpha, \beta)$ and $k \in\{1, \ldots, n\}$ such that

$$
\left|\hat{a}_{k}\left(t_{0}\right)\right|=\max _{1 \leq j \leq n}\left|\hat{a}_{j}\left(t_{0}\right)\right| \neq 0 .
$$

This is possible unless $a \equiv 0$ in which case nothing is to prove. Choose a maximal open interval $I \subseteq(\alpha, \beta)$ containing $t_{0}$ such that

$$
M|I|+\left\|\hat{a}^{\prime}\right\|_{L^{1}(I)} \leq B\left|\hat{a}_{k}\left(t_{0}\right)\right|,
$$

where

$$
M=\max _{1 \leq j \leq n}\left(\operatorname{Lip}_{I}\left(a_{j}^{(d-1)}\right)\right)^{1 / d}\left|\hat{a}_{k}\left(t_{0}\right)\right|^{\left(d-d_{j}\right) / d} .
$$

Consider the point $\underline{p}=\underline{a}\left(t_{0}\right)$, where $\underline{a}$ is the curve defined in (5.3). By (8.2), $\underline{p}$ is an element of the set $K$ defined in (4.1). By the properties of the uniform slice reduction specified in Section 4.3, the ball $B_{\rho}(\underline{p})$ is contained in some ball of the finite cover $\mathcal{B}$ of $K$. By Lemma 5.4 and (8.1), the length of the curve $\left.\underline{a}\right|_{I}$ is bounded by $\rho$. Thus

$$
b \in C^{d-1,1}(\bar{I}, \tau(W)) \text { is obtained by Lemma } 4.4 \text { and satisfies (6.2). }
$$


Lemma 8.1. Assume that $V^{G}=\{0\}$. Let $(\alpha, \beta) \subseteq \mathbb{R}$ be a bounded open interval and let $a \in C^{d-1,1}([\alpha, \beta], \sigma(V))$. Let $B$ be a positive constant satisfying $(8.1)$. Let $t_{0} \in(\alpha, \beta)$ and $k \in$ $\{1, \ldots, n\}$ be such that (8.2) holds. Let $I$ be an open interval with $t_{0} \in I \subseteq(\alpha, \beta)$ satisfying (8.3) and $b$ the reduced curve from (8.5). Then $\left(\left.a\right|_{I}, I, t_{0}, k ; b\right)$ is reduced admissible data for $G \circlearrowleft V$.

Proof. It remains to prove $(6.1)$, i.e., for all $j=1, \ldots, n$ and $s=1, \ldots, d-1$,

$$
\begin{aligned}
& \left\|a_{j}^{(s)}\right\|_{L^{\infty}(I)} \leq C|I|^{-s}\left|\hat{a}_{k}\left(t_{0}\right)\right|^{d_{j}}, \\
& \operatorname{Lip}_{I}\left(a_{j}^{(d-1)}\right) \leq C|I|^{-d}\left|\hat{a}_{k}\left(t_{0}\right)\right|^{d_{j}},
\end{aligned}
$$

for $C=C(G \circlearrowleft V)$. The second bound is immediate from (8.3). Let $t \in I$. By Lemma 5.1,

$$
\left|a_{j}^{(s)}(t)\right| \leq C|I|^{-s}\left(V_{I}\left(a_{j}\right)+V_{I}\left(a_{j}\right)^{(d-s) / d} \operatorname{Lip}_{I}\left(a_{j}^{(d-1)}\right)^{s / d}|I|^{s}\right) .
$$

By (5.6) (it is clear that $\left(\left.a\right|_{I}, I, t_{0}, k\right)$ is admissible data for $\left.G \circlearrowleft V\right)$,

$$
V_{I}\left(a_{j}\right) \leq 2\left\|a_{j}\right\|_{L^{\infty}(I)} \leq 2(4 / 3)^{d}\left|\hat{a}_{k}\left(t_{0}\right)\right|^{d_{j}},
$$

and, by (8.3),

$$
\max _{1 \leq j \leq n}\left(\operatorname{Lip}_{I}\left(a_{j}^{(d-1)}\right)\right)^{s / d}\left|\hat{a}_{k}\left(t_{0}\right)\right|^{-d_{j} s / d}|I|^{s}=\left|\hat{a}_{k}\left(t_{0}\right)\right|^{-s} M^{s}|I|^{s} \leq 1 .
$$

Thus

$$
\left|a_{j}^{(s)}(t)\right| \leq C|I|^{-s}\left|\hat{a}_{k}\left(t_{0}\right)\right|^{d_{j}}\left(C_{1}+C_{2} \operatorname{Lip}_{I}\left(a_{j}^{(d-1)}\right)^{s / d}\left|\hat{a}_{k}\left(t_{0}\right)\right|^{-d_{j} s / d}|I|^{s}\right) \leq C_{3}|I|^{-s}\left|\hat{a}_{k}\left(t_{0}\right)\right|^{d_{j}},
$$

for constants $C_{i}$ that depend only on $d$. So (6.1) is proved.

\section{Step 2: The inductive argument}

The heart of the proof of Theorem 1.1 is the following

Proposition 8.2. Let $\left(a, I, t_{0}, k ; b\right)$ be reduced admissible data for $G \circlearrowleft V$. Then every continuous lift $\bar{b} \in C^{0}(I, W)$ of $b$ is absolutely continuous and satisfies

$$
\left\|\bar{b}^{\prime}\right\|_{L^{p}(I)} \leq C\left(\left\||I|^{-1}\left|\hat{a}_{k}\left(t_{0}\right)\right|\right\|_{L^{p}(I)}+\left\|\hat{b}^{\prime}\right\|_{L^{p}(I)}\right),
$$

for all $1 \leq p<d^{\prime}$ and a constant $C$ depending only on $G \circlearrowleft V$ and $p$.

Remark 8.3. Notice that we bound the $L^{p}$-norm of the derivative of a general lift $\bar{b}$ by the $L^{p}$-norm of the derivatives of the lifts $\hat{b}_{i}$ for the standard action of rotation in $\mathbb{C}$.

Proof of Proposition 8.2. We proceed by induction on the group order.

Induction basis. Proposition 8.2 trivially holds, if the slice representation $H \circlearrowleft W$ is trivial. In that case $\mathbb{C}[W]^{H} \cong \mathbb{C}[W]$ and any system $\tau=\left(\tau, \ldots, \tau_{m}\right)$ of linear coordinates is a minimal system of generators. Hence $\tau: W \rightarrow \mathbb{C}^{m}$ is a linear isomorphism. Moreover, $e_{1}=e_{2}=\cdots=$ $e_{m}=e=1$ whence $\hat{b}_{i}=b_{i}$ for all $i$. Any lift of $b \in C^{d-1,1}(\bar{I}, \tau(W))$ is of the form $\bar{b}=\tau^{-1} \circ b$ and thus (8.6) is trivially satisfied.

Inductive step. Let us set

$$
I^{\prime}:=I \backslash\{t \in I: b(t)=0\} .
$$


For each $t_{1} \in I^{\prime}$ choose $\ell \in\{1, \ldots, m\}$ such that (6.9) holds. By Section 6.4, there is an open interval $J=J\left(t_{1}\right), t_{1} \in J \subseteq I^{\prime}$, such that (6.11), i.e.,

$$
|J||I|^{-1}\left|\hat{a}_{k}\left(t_{0}\right)\right|+\left\|\hat{b}^{\prime}\right\|_{L^{1}(J)}=D\left|\hat{b}_{\ell}\left(t_{1}\right)\right| .
$$

The constant $D$ can be chosen sufficiently small such that the length of the curve $\left.\underline{b}\right|_{J}$ is bounded by the uniform reduction radius $\sigma$ of the representation $H \circlearrowleft W$. It suffices to take

$$
D<\min \left\{\frac{1}{3}, \frac{\sigma}{3 e^{2} 2^{e}}, C_{1}^{-1}\right\}
$$

where $C_{1}$ is the constant in (6.10). This follows from Lemma 6.4 and the arguments in Section 4.3 and in Step 1 applied to $b$.

Then Lemma 4.4 provides a curve $c \in C^{d-1,1}(\bar{J}, \pi(X))$, where $K \circlearrowleft X$ is a slice representation of $H \circlearrowleft W, \pi=\left(\pi_{1}, \ldots, \pi_{q}\right)$ is a system of homogeneous basic invariants with degrees $f_{1}, \ldots, f_{q}$, and $f=\max _{h} f_{h}$. The components of $c$ satisfy

$$
c_{h}=b_{\ell}^{f_{h} / e_{\ell}} \theta_{h}\left(b_{\ell}^{-e_{1} / e_{\ell}} b_{1}, \ldots, b_{\ell}^{-e_{m} / e_{\ell}} b_{m}\right), \quad h=1, \ldots, q,
$$

for suitable analytic functions $\theta_{h}$. We adopt our usual convention that

$$
\hat{c}_{h}: J \rightarrow \mathbb{C}
$$

denotes a fixed continuous selection of $c_{h}^{1 / f_{h}}$ and set

$$
\hat{c}=\left(\hat{c}_{1}, \ldots, \hat{c}_{q}\right): J \rightarrow \mathbb{C}^{q} .
$$

In view of Lemma 6.4 we conclude that $\left(b, J, t_{1}, \ell ; c\right)$ is reduced admissible data for $H \circlearrowleft W$.

By Proposition 7.1 (where (6.11) plays the role of (7.1)), we may conclude that there is a countable family $\left\{\left(J_{\gamma}, t_{\gamma}, \ell_{\gamma}, c_{\gamma}\right)\right\}$ of open intervals $J_{\gamma} \subseteq I^{\prime}$, of points $t_{\gamma} \in J_{\gamma}$, of integers $\ell_{\gamma} \in\{1, \ldots, m\}$, and reduced curves $c_{\gamma}$ such that, for all $\gamma$,

- $\left(b, J_{\gamma}, t_{\gamma}, \ell_{\gamma} ; c_{\gamma}\right)$ is reduced admissible data for $H \circlearrowleft W$,

- we have

$$
\left|J_{\gamma}\right||I|^{-1}\left|\hat{a}_{k}\left(t_{0}\right)\right|+\left\|\hat{b}^{\prime}\right\|_{L^{1}\left(J_{\gamma}\right)}=D\left|\hat{b}_{\ell_{\gamma}}\left(t_{\gamma}\right)\right|
$$

- and

$$
\bigcup_{\gamma} J_{\gamma}=I^{\prime}, \quad \sum_{\gamma}\left|J_{\gamma}\right| \leq 2\left|I^{\prime}\right|
$$

Let $\bar{b} \in C^{0}(I, W)$ be a continuous lift of $b$. Fix $\gamma$ and let $K \circlearrowleft X$ be the corresponding slice representation of $H \circlearrowleft W$. Since $H$ is a finite group, we have $W \cong X$. With this identification and the decomposition $X=X^{K} \oplus X^{\prime}$ we may deduce that the component of $\bar{b}$ in $X^{\prime}$ is a continuous lift of $c_{\gamma}$ on the interval $J_{\gamma}$. To simplify the notation we will assume without loss of generality that $X^{K}=\{0\}$ and that $\bar{b}$ is a lift of $c_{\gamma}$ on the interval $J_{\gamma}$.

The induction hypothesis implies that $\bar{b}$ is absolutely continuous on $J_{\gamma}$ and satisfies

$$
\left\|\bar{b}^{\prime}\right\|_{L^{p}\left(J_{\gamma}\right)} \leq C\left(\left\|\left|J_{\gamma}\right|^{-1}\left|\hat{b}_{\ell_{\gamma}}\left(t_{\gamma}\right)\right|\right\|_{L^{p}\left(J_{\gamma}\right)}+\left\|\hat{c}_{\gamma}^{\prime}\right\|_{L^{p}\left(J_{\gamma}\right)}\right)
$$

for all $1 \leq p<e^{\prime}$, where $C$ is a constant depending only on $H \circlearrowleft W$ and $p$. 


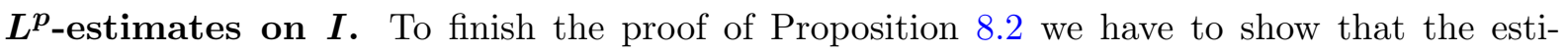
mates (8.10) on the subintervals $J_{\gamma}$ imply the bound (8.6) on $I$. To this end we observe that Corollary 6.3 (applied to $\left.\left(b, J_{\gamma}, t_{\gamma}, \ell_{\gamma} ; c_{\gamma}\right)\right)$ implies that, for all $p$ with $1 \leq p<f_{\gamma}^{\prime}$,

$$
\left\|\hat{c}_{\gamma}^{\prime}\right\|_{L^{p}\left(J_{\gamma}\right)}^{*} \leq C\left|J_{\gamma}\right|^{-1}\left|\hat{b}_{\ell_{\gamma}}\left(t_{\gamma}\right)\right|
$$

for a constant $C$ that depends only on $H \circlearrowleft W$ and $p$.

Now (8.11) and (8.8) allow us to estimate the right-hand side of (8.10):

$$
\begin{aligned}
\left\|\left|J_{\gamma}\right|^{-1}\left|\hat{b}_{\ell_{\gamma}}\left(t_{\gamma}\right)\right|\right\|_{L^{p}\left(J_{\gamma}\right)}^{*}+\left\|\hat{c}_{\gamma}^{\prime}\right\|_{L^{p}\left(J_{\gamma}\right)}^{*} & =\left|J_{\gamma}\right|^{-1}\left|\hat{b}_{\ell_{\gamma}}\left(t_{\gamma}\right)\right|+\left\|\hat{c}_{\gamma}^{\prime}\right\|_{L^{p}\left(J_{\gamma}\right)}^{*} \\
& \leq C\left|J_{\gamma}\right|^{-1}\left|\hat{b}_{\ell_{\gamma}}\left(t_{\gamma}\right)\right| \\
& =C D^{-1}\left(\left\||I|^{-1}\left|\hat{a}_{k}\left(t_{0}\right)\right|\right\|_{L^{1}\left(J_{\gamma}\right)}^{*}+\left\|\hat{b}^{\prime}\right\|_{L^{1}\left(J_{\gamma}\right)}^{*}\right) \\
& \leq C D^{-1}\left(\left\||I|^{-1}\left|\hat{a}_{k}\left(t_{0}\right)\right|\right\|_{L^{p}\left(J_{\gamma}\right)}^{*}+\left\|\hat{b}^{\prime}\right\|_{L^{p}\left(J_{\gamma}\right)}^{*}\right)
\end{aligned}
$$

and therefore

$$
\left\|\left|J_{\gamma}\right|^{-1}\left|\hat{b}_{\ell_{\gamma}}\left(t_{\gamma}\right)\right|\right\|_{L^{p}\left(J_{\gamma}\right)}^{p}+\left\|\hat{c}_{\gamma}^{\prime}\right\|_{L^{p}\left(J_{\gamma}\right)}^{p} \leq C D^{-p}\left(\left\||I|^{-1}\left|\hat{a}_{k}\left(t_{0}\right)\right|\right\|_{L^{p}\left(J_{\gamma}\right)}^{p}+\left\|\hat{b}^{\prime}\right\|_{L^{p}\left(J_{\gamma}\right)}^{p}\right),
$$

for a constant $C$ that depends only on $H \circlearrowleft W$ and $p$.

Let us now glue the bounds on $J_{\gamma}$ to a bound on $I$. By (8.9), (8.10), and (8.12),

$$
\sum_{\gamma}\left\|\bar{b}^{\prime}\right\|_{L^{p}\left(J_{\gamma}\right)}^{p} \leq C D^{-p}\left(\left\||I|^{-1}\left|\hat{a}_{k}\left(t_{0}\right)\right|\right\|_{L^{p}(I)}^{p}+\left\|\hat{b}^{\prime}\right\|_{L^{p}(I)}^{p}\right),
$$

for a constant $C$ that depends only on $H \circlearrowleft W$ and $p$. Thus $\bar{b}$ is absolutely continuous on $I^{\prime}$ and

$$
\left\|\bar{b}^{\prime}\right\|_{L^{p}\left(I^{\prime}\right)} \leq C D^{-1}\left(\left\||I|^{-1}\left|\hat{a}_{k}\left(t_{0}\right)\right|\right\|_{L^{p}(I)}+\left\|\hat{b}^{\prime}\right\|_{L^{p}(I)}\right),
$$

for a constant $C$ that depends only on $H \circlearrowleft W$ and $p$. Since $\bar{b}$ vanishes on $I \backslash I^{\prime}$, Lemma 2.1 implies that $\bar{b}$ is absolutely continuous on $I$ and satisfies (8.6), since $D=D(H \circlearrowleft W)$ by (8.7). This completes the proof of Proposition 8.2.

\section{Step 3: The proof of Theorem 1.1}

In view of Lemma 4.1 we may assume $V^{G}=\{0\}$. Let $a \in C^{d-1,1}([\alpha, \beta], \sigma(V))$. Suppose that $B$ is a positive constant fulfilling (8.1) and assume that $t_{0} \in(\alpha, \beta), k \in\{1, \ldots, n\}$, and $I \ni t_{0}$ satisfy (8.2) and (8.3). Let $b \in C^{d-1,1}(\bar{I}, \tau(W))$ be the reduced curve from (8.5). Then Lemma 8.1 implies that $\left(a, I, t_{0}, k ; b\right)$ is reduced admissible data and consequently each continuous lift $\bar{b}$ of $b$ satisfies (8.6), by Proposition 8.2. In particular, if $\bar{a} \in C^{0}((\alpha, \beta), V)$ is a continuous lift of $a$, then we may assume that $\left.\bar{a}\right|_{I}$ is a lift of $b$. It follows that $\bar{a}$ is absolutely continuous on $I$ and

$$
\left\|\bar{a}^{\prime}\right\|_{L^{p}(I)} \leq C(G \circlearrowleft V, p)\left(\left\||I|^{-1}\left|\hat{a}_{k}\left(t_{0}\right)\right|\right\|_{L^{p}(I)}+\left\|\hat{b}^{\prime}\right\|_{L^{p}(I)}\right) .
$$

Our next goal is to estimate the right-hand side of (8.13) in terms of $a$.

By Corollary 6.3, we get for all $p$ with $1 \leq p<e^{\prime}$,

$$
\left\||I|^{-1}\left|\hat{a}_{k}\left(t_{0}\right)\right|\right\|_{L^{p}(I)}^{*}+\left\|\hat{b}^{\prime}\right\|_{L^{p}(I)}^{*} \leq C|I|^{-1}\left|\hat{a}_{k}\left(t_{0}\right)\right|,
$$

where the constant $C$ depends only on $G \circlearrowleft V$ and $p$. At this stage we distinguish the two cases of strict inequality or equality in (8.3): 
(i) Strict inequality: we have $I=(\alpha, \beta)$ and

$$
M|I|+\left\|\hat{a}^{\prime}\right\|_{L^{1}(I)}<B\left|\hat{a}_{k}\left(t_{0}\right)\right| .
$$

(ii) Equality:

$$
M|I|+\left\|\hat{a}^{\prime}\right\|_{L^{1}(I)}=B\left|\hat{a}_{k}\left(t_{0}\right)\right| .
$$

Case $(i)$. In this case we can reduce to the curve $b \in C^{d-1,1}(\bar{I}, \tau(W))$ on the whole interval $I=(\alpha, \beta) ;$ cf. Step 1. Thus, (8.14) becomes

$$
\left\|(\beta-\alpha)^{-1}\left|\hat{a}_{k}\left(t_{0}\right)\right|\right\|_{L^{p}((\alpha, \beta))}+\left\|\hat{b}^{\prime}\right\|_{L^{p}((\alpha, \beta))} \leq C(\beta-\alpha)^{-1+1 / p}\left|\hat{a}_{k}\left(t_{0}\right)\right|,
$$

which can be bounded by

$$
C(\beta-\alpha)^{-1+1 / p} \max _{1 \leq j \leq n}\left\|a_{j}\right\|_{L^{\infty}((\alpha, \beta))}^{1 / d_{j}} .
$$

By (8.13), $\bar{a}$ is absolutely continuous on $(\alpha, \beta)$ and

$$
\left\|\bar{a}^{\prime}\right\|_{L^{p}((\alpha, \beta))} \leq C(\beta-\alpha)^{-1+1 / p} \max _{1 \leq j \leq n}\left\|a_{j}\right\|_{L^{\infty}((\alpha, \beta))}^{1 / d_{j}},
$$

where $C=C(G \circlearrowleft V, p)$.

Remark 8.4. The bound in (8.17) tends to infinity if $\beta-\alpha \rightarrow 0$ unless $p=1$.

Case (ii). Using (8.16) to estimate (8.14) (as in the derivation of (8.12)), we get

$$
\left\||I|^{-1}\left|\hat{a}_{k}\left(t_{0}\right)\right|\right\|_{L^{p}(I)}+\left\|\hat{b}^{\prime}\right\|_{L^{p}(I)} \leq C\left(M\|1\|_{L^{p}(I)}+\left\|\hat{a}^{\prime}\right\|_{L^{p}(I)}\right),
$$

for a constant $C$ that depends only on $G \circlearrowleft V$ and $p$; note that $B=B(G \circlearrowleft V)$ by (8.1). Thus, by $(8.13)$,

$$
\left\|\bar{a}^{\prime}\right\|_{L^{p}(I)} \leq C\left(M\|1\|_{L^{p}(I)}+\left\|\hat{a}^{\prime}\right\|_{L^{p}(I)}\right) .
$$

Let us set $A:=\max _{1 \leq j \leq n}\left\|a_{j}\right\|_{C^{d-1,1}([\alpha, \beta])}^{1 / d_{j}}$. Then

$$
M=\max _{1 \leq j \leq n}\left(\operatorname{Lip}_{I}\left(a_{j}^{(d-1)}\right)\right)^{1 / d}\left|\hat{a}_{k}\left(t_{0}\right)\right|^{\left(d-d_{j}\right) / d} \leq \max _{1 \leq j \leq n} A^{d_{j} / d} A^{\left(d-d_{j}\right) / d}=A .
$$

Consequently,

$$
\left\|\bar{a}^{\prime}\right\|_{L^{p}(I)} \leq C\left(A\|1\|_{L^{p}(I)}+\left\|\hat{a}^{\prime}\right\|_{L^{p}(I)}\right) .
$$

By Proposition 7.1 (applied to $a$ instead of $b$ and (8.16) instead of (7.1)), we can cover the set $(\alpha, \beta) \backslash\{t: a(t)=0\}$ by a countable family $\mathcal{I}$ of open intervals $I$ on which (8.18) holds and such that $\sum_{I \in \mathcal{I}}|I| \leq 2(\beta-\alpha)$. Together with Lemma 2.1 we may conclude that $\bar{a}$ is absolutely continuous on $(\alpha, \beta)$ and satisfies

$$
\left\|\bar{a}^{\prime}\right\|_{L^{p}((\alpha, \beta))} \leq C\left(A\|1\|_{L^{p}((\alpha, \beta))}+\left\|\hat{a}^{\prime}\right\|_{L^{p}((\alpha, \beta))}\right),
$$

Using (3.1) and the fact that $1-1 / d_{j}<1 / p$ for all $j \leq n$, we obtain

$$
\begin{aligned}
& \left\|\bar{a}^{\prime}\right\|_{L^{p}((\alpha, \beta))} \\
& \quad \leq C\left(A(\beta-\alpha)^{1 / p}+\sum_{j=1}^{n} \max \left\{\left(\operatorname{Lip}_{(\alpha, \beta)}\left(a_{j}^{\left(d_{j}-1\right)}\right)\right)^{1 / d_{j}}(\beta-\alpha)^{1-1 / d_{j}},\left\|a_{j}^{\prime}\right\|_{L^{\infty}((\alpha, \beta))}^{1 / d_{j}}\right\}\right) \\
& \quad \leq C \max \left\{1,(\beta-\alpha)^{1 / p}\right\} \max _{1 \leq j \leq n}\left\|a_{j}\right\|_{C^{d-1,1}([\alpha, \beta])}^{1 / d_{j}},
\end{aligned}
$$

where $C=C(G \circlearrowleft V, p)$. The proof of Theorem 1.1 is complete. 


\section{Proof of Remark 1.2}

Remark 1.2(a) is clear by the above discussion.

Suppose that there exists $s \in[\alpha, \beta]$ such that $a(s)=0$. Then for all $t \in(\alpha, \beta)$ and all $j$,

$$
\left|\hat{a}_{j}(t)\right|=\left|\int_{s}^{t} \hat{a}_{j}^{\prime}(\tau) \mathrm{d} \tau\right| \leq\left\|\hat{a}_{j}^{\prime}\right\|_{L^{1}((\alpha, \beta))}
$$

Thus the Case $(i)$, i.e., (8.15), cannot occur. This implies Remark 1.2(b).

If the representation is coregular, then $\sigma(V)=\mathbb{C}^{n}$ and we may use a simple version of Whitney's extension theorem to extend $a$ to a curve defined on $(\alpha-1, \beta+1)$ which vanishes at the endpoints of this larger interval and such that $\|a\|_{C^{d-1,1}([\alpha-1, \beta+1])} \leq C\|a\|_{C^{d-1,1}([\alpha, \beta])}$, where $C$ is a universal constant independent of $(\alpha, \beta)$. As above one sees that Case $(i)$ cannot occur and hence we obtain the bound (1.1) with the constant (1.2) on the larger interval $(\alpha-1, \beta+1)$. Thanks to the continuity of the extension, we obtain the desired bound on the original interval $(\alpha, \beta)$. For details see [16]. This shows Remark 1.2(c). In general, if $\sigma(V)$ is a proper subset of $\mathbb{C}^{n}$, it is not clear that the extended curve is contained in $\sigma(V)$ and hence liftable.

To see Remark 1.2(d) we observe that under the assumption that $a^{(j)}(\alpha)=a^{(j)}(\beta)=0$ for all $j=1, \ldots, d-1$ the curve $a$ can be extended beyond the interval $(\alpha, \beta)$ by setting $a(t)=a(\alpha)$ for $t<\alpha$ and $a(t)=a(\beta)$ for $t>\beta$. Then the extended curve still lies in $\sigma(V)$ and we have $\left\|a_{j}\right\|_{C^{d-1,1}([\alpha-1, \beta+1])}=\left\|a_{j}\right\|_{C^{d-1,1}([\alpha, \beta])}$ for all $j=1, \ldots, n$. Choose a smooth function $\varphi: \mathbb{R} \rightarrow[0,1]$ such that $\varphi(t)=1$ for $t \leq 0$ and $\varphi(t)=0$ for $t \geq 1$. Then $\psi(t):=\varphi(\alpha-t) \varphi(t-\beta)$ is equal to 1 on $[\alpha, \beta]$ and 0 outside $[\alpha-1, \beta+1]$. Let us consider

$$
a_{\psi}:=\left(\psi^{d_{1}} a_{1}, \psi^{d_{2}} a_{2}, \ldots, \psi^{d_{n}} a_{n}\right),
$$

which is a $C^{d-1,1}$-curve in $\sigma(V)$ coinciding with $a$ on $[\alpha, \beta]$ and vanishing at the endpoints of $[\alpha-1, \beta+1]$. As above we conclude that for $a_{\psi}$ Case $(i)$ cannot occur. Since we have

$$
\left\|\left(a_{\psi}\right)_{j}\right\|_{C^{d-1,1}([\alpha-1, \beta+1])} \leq C(\varphi)\left\|a_{j}\right\|_{C^{d-1,1}([\alpha, \beta])}, \quad j=1, \ldots, n,
$$

it is easy to conclude Remark $1.2(d)$.

\section{Proof of Theorem 1.4}

Lemma 9.1. Let $c: I \rightarrow \sigma(V)$ be continuous and let $\bar{c}: J \rightarrow V$ a continuous lift of $c$ on an open proper subinterval $J \Subset I$ of $I$. Then $\bar{c}$ can be extended to a continuous lift of $c$ defined on $I$.

Proof. Since we already know that $c$ admits a continuous lift $\bar{c}_{1}$ on $I$ it suffices to show that $\bar{c}$ extends continuously to the endpoints of $J$. Then $\bar{c}$ can be extended left and right of $J$ by $\bar{c}_{1}$ after applying a fixed transformation from $G$.

So let $t_{0}$ be the (say) right endpoint of $J$. The set of limit points $A$ of $\bar{c}(t)$ as $t \rightarrow t_{0}^{-}$is contained in the orbit corresponding to $c\left(t_{0}\right)$. On the other hand $A$ must be connected, by the continuity of $\bar{c}$. Since every orbit is finite, $A$ consists of just one point.

Lemma 9.2. Let $\bar{c}_{1}, \bar{c}_{2}$ be continuous lifts of a curve $c: I \rightarrow \sigma(V)$. If $\bar{c}_{1}$ is absolutely continuous and $\bar{c}_{1} \in W^{1, p}(I)$, then $\bar{c}_{2}$ is absolutely continuous, $\bar{c}_{2} \in W^{1, p}(I)$, and

$$
\left\|\bar{c}_{2}^{\prime}\right\|_{L^{p}(I)} \leq C\left\|\bar{c}_{1}^{\prime}\right\|_{L^{p}(I)},
$$

where $C$ depends only on $G \circlearrowleft V$ and on the coordinate system on $V$. 
Proof. For each subset $E$ of $I$ we have $\bar{c}_{2}(E) \subseteq \bigcup_{g \in G} g \bar{c}_{1}(E)$. It follows that

$$
\operatorname{length}\left(\bar{c}_{2}\right) \leq \sum_{g \in G} \operatorname{length}\left(g \bar{c}_{1}\right)<\infty
$$

and that $\bar{c}_{2}$ has the Luzin $(\mathrm{N})$ property. Hence $\bar{c}_{2}$ is absolutely continuous.

Suppose that both $\bar{c}_{1}$ and $\bar{c}_{2}$ are differentiable at $t$. After replacing $\bar{c}_{1}$ with $g \bar{c}_{1}$ for a suitable $g \in G$ we may suppose that $\bar{c}_{1}(t)=\bar{c}_{2}(t)=: v$. Then after switching to the slice representation at $v$ we have, for $g_{h} \in G_{v}$ (which entails $\bar{c}_{2}(t)=g_{h} \bar{c}_{1}(t)$ ),

$$
\frac{\bar{c}_{2}(t+h)-\bar{c}_{2}(t)}{h}=\frac{g_{h} \bar{c}_{1}(t+h)-g_{h} \bar{c}_{1}(t)}{h}=g_{h}\left(\frac{\bar{c}_{1}(t+h)-\bar{c}_{1}(t)}{h}\right),
$$

which implies that $\bar{c}_{2}^{\prime}(t) \in G_{v} \bar{c}_{1}^{\prime}(t)$, since $G$, and hence $G_{v}$, is finite. This implies the lemma.

Now we are ready to prove Theorem 1.4.

Let $\bar{f} \in C^{0}(U, V)$ be a continuous lift of $f \in C^{d-1,1}(\bar{\Omega}, \sigma(V))$ on $U$.

By Theorem 1.1, $\bar{f}$ is absolutely continuous along affine lines parallel to the coordinate axes (restricted to $U$ ). So $\bar{f}$ possesses partial derivatives of first order which are defined almost everywhere and measurable.

Set $x=(t, y)$, where $t=x_{1}, y=\left(x_{2}, \ldots, x_{m}\right)$, and let $U_{1}$ be the orthogonal projection of $U$ on the hyperplane $\left\{x_{1}=0\right\}$. For each $y \in U_{1}$ we denote by $U^{y}:=\{t \in \mathbb{R}:(t, y) \in U\}$ the corresponding section of $U$.

Let $\bar{f}^{y}(t):=\bar{f}(t, y)$ for $t \in U^{y}$; it is clear that $\bar{f}^{y}$ is a continuous lift of $\left.f\right|_{U^{y} \times\{y\}}$. Recall that $\Omega=I_{1} \times \cdots \times I_{m}$ is an open box in $\mathbb{R}^{m}$. Let $\mathcal{C}^{y}$ denote the set of connected components $J$ of the open subset $U^{y} \subseteq \mathbb{R}$. For each $J \in \mathcal{C}^{y}$ we may extend the lift $\bar{f}^{y}$ continuously to $I_{1} \times\{y\}$, by Lemma 9.1. So for each $J \in \mathcal{C}^{y}$ we get a continuous lift $\bar{f}_{J}^{y}$ of $\left.f\right|_{I_{1} \times\{y\}}$ such that $\left.\bar{f}_{J}^{y}\right|_{J}=\left.\bar{f}^{y}\right|_{J}$.

By Theorem 1.1, for all $y \in U_{1}$ and $J \in \mathcal{C}^{y}$, the lift $\bar{f}_{J}^{y}$ is absolutely continuous on $I_{1}$ with $\left(\bar{f}_{J}^{y}\right)^{\prime} \in L^{p}\left(I_{1}\right)$, for $1 \leq p<d /(d-1)$, and

$$
\left\|\left(\bar{f}_{J}^{y}\right)^{\prime}\right\|_{L^{p}\left(I_{1}\right)} \leq C \max _{1 \leq i \leq n}\left\|f_{i}\right\|_{C^{d-1,1}(\bar{\Omega})}^{1 / d_{i}}
$$

where $C$ depends only on $G \circlearrowleft V, p$, and $\left|I_{1}\right|$.

Let $J, J_{0} \in \mathcal{C}^{y}$ be arbitrary. By Lemma 9.2 , both $\left(\bar{f}_{J}^{y}\right)^{\prime}$ and $\left(\bar{f}_{J_{0}}^{y}\right)^{\prime}$ belong to $L^{p}\left(I_{1}\right)$ and

$$
\left\|\left(\bar{f}_{J}^{y}\right)^{\prime}\right\|_{L^{p}(J)} \leq C(G \circlearrowleft V)\left\|\left(\bar{f}_{J_{0}}^{y}\right)^{\prime}\right\|_{L^{p}(J)} .
$$

Thus,

$$
\left\|\left(\bar{f}^{y}\right)^{\prime}\right\|_{L^{p}\left(U^{y}\right)}^{p}=\sum_{J \in \mathcal{C}^{y}}\left\|\left(\bar{f}_{J}^{y}\right)^{\prime}\right\|_{L^{p}(J)}^{p} \leq C^{p} \sum_{J \in \mathcal{C}^{y}}\left\|\left(\bar{f}_{J_{0}}^{y}\right)^{\prime}\right\|_{L^{p}(J)}^{p}=C^{p}\left\|\left(\bar{f}_{J_{0}}^{y}\right)^{\prime}\right\|_{L^{p}\left(U^{y}\right)}^{p}
$$

and consequently, by (9.1),

$$
\left\|\left(\bar{f}^{y}\right)^{\prime}\right\|_{L^{p}\left(U^{y}\right)} \leq C \max _{1 \leq i \leq n}\left\|f_{i}\right\|_{C^{d-1,1}(\bar{\Omega})}^{1 / d_{i}} .
$$

By Fubini's theorem,

$$
\int_{U}\left|\partial_{1} \bar{f}(x)\right|^{p} \mathrm{~d} \mathrm{x}=\int_{U_{1}} \int_{U^{y}}\left|\partial_{1} \bar{f}(t, y)\right|^{p} \mathrm{~d} t \mathrm{~d} y \leq\left(C \max _{1 \leq i \leq n}\left\|f_{i}\right\|_{C^{d-1,1}(\bar{\Omega})}^{1 / d_{i}}\right)^{p} \int_{U_{1}} \mathrm{~d} y .
$$

This implies Theorem 1.4.

For Remark 1.5 notice that, if $G \circlearrowleft V$ is coregular, then $\sigma(V)=V / / G=\mathbb{C}^{n}$ and hence we may use Whitney's extension theorem to extend $f$ to a mapping defined on a box $R$ containing $\Omega$ such that the $C^{d-1,1}$-norm on $R$ is bounded by the $C^{d-1,1}$-norm on $\Omega$ times a constant. In general it is not clear that after extension $f$ still takes values in $\sigma(V)$. 


\section{$10 Q$-valued functions}

The basic reference for the background on $Q$-valued Sobolev functions used in this section is [4].

\subsection{The metric space $\mathcal{A}_{Q}\left(\mathbb{R}^{n}\right)$}

Unordered $Q$-tuples of points in $\mathbb{R}^{n}$ can be formalized as positive atomic measures of mass $Q$. Let $\llbracket p_{i} \rrbracket$ denote the Dirac mass at $p_{i} \in \mathbb{R}^{n}$. We consider the space

$$
\mathcal{A}_{Q}\left(\mathbb{R}^{n}\right):=\left\{\sum_{i=1}^{Q} \llbracket p_{i} \rrbracket: p_{i} \in \mathbb{R}^{n}\right\}
$$

of unordered $Q$-tuples of points in $\mathbb{R}^{n}$. Then $\mathcal{A}_{Q}\left(\mathbb{R}^{n}\right)$ is a complete metric space when endowed with the metric

$$
d\left(\sum_{i} \llbracket p_{i} \rrbracket, \sum_{i} \llbracket q_{i} \rrbracket\right):=\min _{\sigma \in \mathrm{S}_{Q}}\left(\sum_{i}\left|p_{i}-q_{\sigma(i)}\right|^{2}\right)^{1 / 2} .
$$

\subsection{Invariants}

There is a natural one-to-one correspondence between the unordered $Q$-tuples $\sum_{i} \llbracket p_{i} \rrbracket \in \mathcal{A}_{Q}\left(\mathbb{R}^{n}\right)$ and the orbits of the $n$-fold direct sum $W:=\left(\mathbb{R}^{Q}\right)^{\oplus n}$ of the tautological representation $\mathbb{R}^{Q}$ of the symmetric group $\mathrm{S}_{Q}$. By a result of Weyl [21], the algebra $\mathbb{R}[W]^{\mathrm{S}_{Q}}$ is generated by the polarizations of the elementary symmetric functions. Up to integer factors the polarizations are

$$
\begin{aligned}
& \sigma_{1}(u)=\sum_{i} u_{i} \\
& \sigma_{2}(u, v)=\sum_{i \neq j} u_{i} v_{j} \\
& \sigma_{3}(u, v, w)=\sum_{i, j, k \text { all } \neq} u_{i} v_{j} w_{k}, \\
& \ldots \ldots \ldots \ldots \ldots \ldots \ldots \ldots \ldots \ldots \\
& \sigma_{Q}(u, v, \ldots, w)=\sum_{i, j, \ldots, k \text { all } \neq} u_{i} v_{j} \cdots w_{k},
\end{aligned}
$$

where $u=\left(u_{1}, u_{2}, \ldots, u_{Q}\right), v=\left(v_{1}, v_{2}, \ldots, v_{Q}\right)$, etc. A system of generators of $\mathbb{R}[W]^{\mathrm{S}_{Q}}$ is obtained by substituting the arguments $x^{1}, x^{2}, \ldots, x^{n} \in \mathbb{R}^{Q}$ for $u, v, w, \ldots$ in all possible combinations (including repetitions). Note that the $\operatorname{ring} \mathbb{R}[W]^{\mathrm{S}_{Q}}$ is not polynomial unless $n=1$, e.g., by the Shephard-Todd-Chevalley theorem.

\subsection{Subspaces $\mathcal{A}_{G \circlearrowleft \mathbb{R}^{n}}\left(\mathbb{R}^{n}\right)$}

Let $G \circlearrowleft \mathbb{R}^{n}$ be a representation of a finite group $G$. We define the space

$$
\mathcal{A}_{G \circlearrowleft \mathbb{R}^{n}}\left(\mathbb{R}^{n}\right):=\left\{\sum_{g \in G} \llbracket g p \rrbracket: p \in \mathbb{R}^{n}\right\}
$$

of $G$-orbits. It is a closed subspace of the complete metric space $\mathcal{A}_{|G|}\left(\mathbb{R}^{n}\right)$, thus also complete.

A system of generators for $\mathbb{R}[V]^{G}$ can be obtained from the generators of $\mathbb{R}[W]^{\mathrm{S}}|G|$ by means of the Noether map $\eta^{*}: \mathbb{R}[W]^{\mathrm{S}|G|} \rightarrow \mathbb{R}\left[\mathbb{R}^{n}\right]^{G}$, where $\eta: \mathbb{R}^{n} \rightarrow W$ is defined by $\eta(p)(g)=g p$ and $W=\left(\mathbb{R}^{|G|}\right)^{\oplus n}$ is identified with the space of mappings $G \rightarrow \mathbb{R}^{n}$; for details see, e.g., [12]. 


\section{$10.4 Q$-valued Sobolev functions}

Let $\Omega$ be a bounded open subset of $\mathbb{R}^{m}$. A measurable function $f: \Omega \rightarrow \mathcal{A}_{Q}\left(\mathbb{R}^{n}\right)$ is said to be in the Sobolev class $W^{1, p}$ (for $1 \leq p \leq \infty$ ) if

1) $x \mapsto \mathrm{d}(f(x), P) \in W^{1, p}(\Omega)$ for all $P \in \mathcal{A}_{Q}\left(\mathbb{R}^{n}\right)$,

2) there exist functions $\varphi_{1}, \ldots, \varphi_{m} \in L^{p}\left(\Omega, \mathbb{R}^{+}\right)$such that

$$
\left|\partial_{j} \mathrm{~d}(f, P)\right| \leq \varphi_{j} \quad \text { a.e. } \quad \text { in } \quad \Omega \quad \text { for all } \quad P \in \mathcal{A}_{Q}\left(\mathbb{R}^{n}\right) \quad \text { and } \quad j=1, \ldots, m \text {. }
$$

The minimal functions $\varphi_{j}$ satisfying (2) are denoted by $\left|\partial_{j} f\right|$ and they are characterized as follows: for every countable dense subset $\left\{P_{\ell}\right\}_{\ell \in \mathbb{N}} \subseteq \mathcal{A}_{Q}\left(\mathbb{R}^{n}\right)$ and all $j=1, \ldots, m$ we have

$$
\left|\partial_{j} f\right|=\sup _{\ell \in \mathbb{N}}\left|\partial_{j} \mathrm{~d}\left(f, P_{\ell}\right)\right| \quad \text { a.e. } \quad \text { in } \quad \Omega .
$$

One sets $|D f|:=\left(\sum_{j=1}^{m}\left|\partial_{j} f\right|^{2}\right)^{1 / 2}$. This intrinsic approach is developed in [4].

Alternatively, one may use Almgren's extrinsic approach [1] to $Q$-valued Sobolev functions. There is an injective Lipschitz map $\xi: \mathcal{A}_{Q}\left(\mathbb{R}^{n}\right) \rightarrow \mathbb{R}^{N}$, where $N=N(Q, n)$, with Lipschitz constant $\operatorname{Lip}(\xi) \leq 1$ such that the inverse $\theta:=\left.\xi\right|_{\xi\left(\mathcal{A}_{Q}\left(\mathbb{R}^{n}\right)\right)} ^{-1}$ is Lipschitz with Lipschitz constant $\leq C(Q, n)$. Here the constants $N$ and $C$ depend only upon $Q$ and $n$. The inverse $\theta: \xi\left(\mathcal{A}_{Q}\left(\mathbb{R}^{n}\right)\right) \rightarrow \mathcal{A}_{Q}\left(\mathbb{R}^{n}\right)$ has a Lipschitz extension $\Theta: \mathbb{R}^{N} \rightarrow \mathcal{A}_{Q}\left(\mathbb{R}^{n}\right)$. It follows that $\rho:=\xi \circ \Theta$ is a Lipschitz retraction of $\mathbb{R}^{N}$ onto $\xi\left(\mathcal{A}_{Q}\left(\mathbb{R}^{n}\right)\right)$.

A function $f: \Omega \rightarrow \mathcal{A}_{Q}\left(\mathbb{R}^{n}\right)$ is of class $W^{1, p}$ if and only if $\xi \circ f$ belongs to $W^{1, p}\left(\Omega, \mathbb{R}^{N}\right)$, and in that case

$$
|D(\xi \circ f)| \leq|D f| \leq C(Q, n)|D(\xi \circ f)|,
$$

see [4, Theorem 2.4].

\section{5 $Q$-valued Sobolev functions and invariant theory}

We may identify the $\mathrm{S}_{Q}$-module $W=\left(\mathbb{R}^{Q}\right)^{\oplus n}$ with the space of $Q \times n$ matrices $\mathbb{R}^{Q \times n}$. Then $\sigma \in \mathrm{S}_{Q}$ acts on a $Q \times n$ matrix by permuting the rows. Consider the surjective mapping $\pi: \mathbb{R}^{Q \times n} \rightarrow \mathcal{A}_{Q}\left(\mathbb{R}^{n}\right)$ which sends a matrix with rows $p_{1}, \ldots, p_{Q}$ to $\sum_{i=1}^{Q} \llbracket p_{i} \rrbracket$. If we endow $\mathbb{R}^{Q \times n}$ with the Frobenius norm (i.e., $\left.\left\|\left(p_{i j}\right)_{i j}\right\|=\left(\sum_{i=1}^{Q} \sum_{j=1}^{n}\left|p_{i j}\right|^{2}\right)^{1 / 2}\right)$ then $\pi$ is Lipschitz with $\operatorname{Lip}(\pi) \leq 1$

Let $\sigma_{1}, \ldots, \sigma_{r}$ be any system of homogeneous generators of $\mathbb{R}[W]^{\mathrm{S}_{Q}}$. The corresponding map $\sigma=\left(\sigma_{1}, \ldots, \sigma_{r}\right)$ induces a bijective map $\Sigma: \mathcal{A}_{Q}\left(\mathbb{R}^{n}\right) \rightarrow \sigma(W) \subseteq \mathbb{R}^{r}$ such that $\sigma=\Sigma \circ \pi$. We may assume that $d_{j}:=\operatorname{deg} \sigma_{j} \leq Q$ for all $j=1, \ldots, r$.

Theorem 10.1. Let $\Omega$ be a bounded open subset of $\mathbb{R}^{m}$. Let $f: \Omega \rightarrow \mathcal{A}_{Q}\left(\mathbb{R}^{n}\right)$ be continuous. If $\Sigma \circ f \in C^{Q-1,1}\left(\bar{\Omega}, \mathbb{R}^{r}\right)$, then for each relatively compact open $\Omega^{\prime} \subseteq \Omega$ we have $f \in W^{1, \infty}\left(\Omega^{\prime}, \mathcal{A}_{Q}\left(\mathbb{R}^{n}\right)\right)$. Moreover,

$$
\|D f\|_{L^{\infty}\left(\Omega^{\prime}\right)} \leq C\left(Q, n, m, \Omega, \Omega^{\prime}\right)\left(1+\max _{1 \leq j \leq r}\left\|\Sigma_{j} \circ f\right\|_{C^{Q-1,1}(\bar{\Omega})}^{1 / d_{j}}\right) .
$$

Proof. Let us first consider the case that $m=1$ and $\Omega$ is an interval. In that case we even obtain a global statement with $I:=\Omega^{\prime}=\Omega$. Indeed, the curve $c:=\Sigma \circ f$ in $\sigma(W) \subseteq \mathbb{R}^{r}$ admits an absolutely continuous lift $\bar{c}$ to $W$ which belongs to $W^{1, \infty}(I, W)$, by Theorem 1.9. Then the 
statement follows by superposition with the Lipschitz map $\xi \circ \pi$. The uniform bound easily follows from the bound in Theorem 1.9 and (10.2):

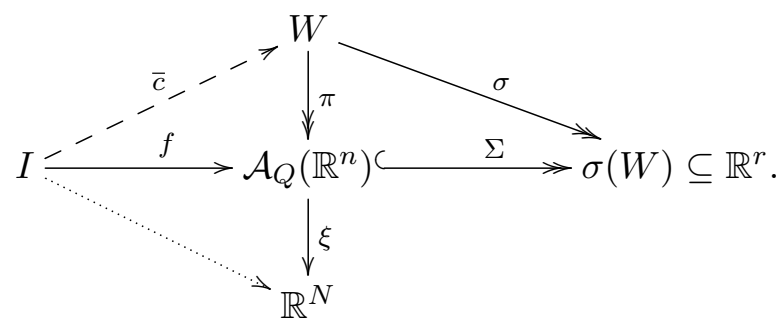

The general case follows from a standard argument by covering $\Omega^{\prime}$ by boxes contained in $\Omega$ and using Fubini's theorem in a similar fashion as in the proof of Theorem 1.4.

Corollary 10.2. The bijective mapping $\Sigma$ induces a bounded mapping

$$
\left(\Sigma^{-1}\right)_{*}: C^{Q-1,1}(\Omega, \sigma(W)) \rightarrow W_{\mathrm{loc}}^{1, \infty}\left(\Omega, \mathcal{A}_{Q}\left(\mathbb{R}^{n}\right)\right), \quad \varphi \mapsto \Sigma^{-1} \circ \varphi .
$$

Proof. It suffices to check that $\Sigma^{-1} \circ \varphi$ is continuous. This follows from the fact that $\pi$ is continuous and that $\sigma$ is proper and thus closed.

\subsection{Multi-valued Sobolev liftings}

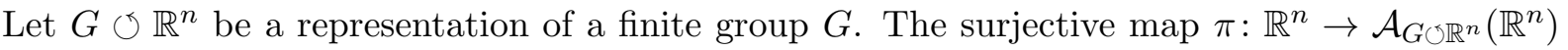
defined by $\pi(p)=\sum_{g \in G} \llbracket g p \rrbracket$ is clearly Lipschitz. Let $\sigma_{1}, \ldots, \sigma_{r}$ be any system of homogeneous generators of $\mathbb{R}\left[\mathbb{R}^{n}\right]^{G}$. There is a bijective map $\Sigma: \mathcal{A}_{G \circlearrowleft \mathbb{R}^{n}}\left(\mathbb{R}^{n}\right) \rightarrow \sigma\left(\mathbb{R}^{n}\right) \subseteq \mathbb{R}^{r}$ such that $\sigma=$ $\Sigma \circ \pi$, since $\sigma=\left(\sigma_{1}, \ldots, \sigma_{r}\right)$ separates orbits. Let $d:=\max _{j} \operatorname{deg} \sigma_{j}$.

Let $\Omega$ be a bounded open subset of $\mathbb{R}^{m}$. We say that a function $f: \Omega \rightarrow \mathcal{A}_{G \circlearrowleft \mathbb{R}^{n}\left(\mathbb{R}^{n}\right)}$ is of class $W^{1, p}$, and write $f \in W^{1, p}\left(\Omega, \mathcal{A}_{G \circlearrowleft \mathbb{R}^{n}}\left(\mathbb{R}^{n}\right)\right)$, if $f \in W^{1, p}\left(\Omega, \mathcal{A}_{|G|}\left(\mathbb{R}^{n}\right)\right)$.

Thus we obtain, analogously to Theorem 10.1,

Theorem 10.3. Let $f: \Omega \rightarrow \mathcal{A}_{G \circlearrowleft \mathbb{R}^{n}}\left(\mathbb{R}^{n}\right)$ be continuous. If $\Sigma \circ f \in C^{d-1,1}\left(\bar{\Omega}, \mathbb{R}^{r}\right)$, then for each relatively compact open $\Omega^{\prime} \subseteq \Omega$ we have $f \in W^{1, \infty}\left(\Omega^{\prime}, \mathcal{A}_{G \circlearrowleft \mathbb{R}^{n}}\left(\mathbb{R}^{n}\right)\right)$. Moreover,

$$
\|D f\|_{L^{\infty}\left(\Omega^{\prime}\right)} \leq C\left(d, n, m, \Omega, \Omega^{\prime}\right)\left(1+\max _{1 \leq j \leq r}\left\|\Sigma_{j} \circ f\right\|_{C^{d-1,1}(\bar{\Omega})}^{1 / d_{j}}\right) .
$$

Corollary 10.4. The bijective mapping $\Sigma$ induces a bounded mapping

$$
\left(\Sigma^{-1}\right)_{*}: C^{d-1,1}\left(\Omega, \sigma\left(\mathbb{R}^{n}\right)\right) \rightarrow W_{\text {loc }}^{1, \infty}\left(\Omega, \mathcal{A}_{G \circlearrowleft \mathbb{R}^{n}}\left(\mathbb{R}^{n}\right)\right), \quad \varphi \mapsto \Sigma^{-1} \circ \varphi .
$$

\subsection{Complex $Q$-valued functions}

It is evident that one can define the space $\mathcal{A}_{Q}\left(\mathbb{C}^{n}\right)$ of unordered $Q$-tuples of points in $\mathbb{C}^{n}$ in analogy to $\mathcal{A}_{Q}\left(\mathbb{R}^{n}\right)$. It is a complete metric space with the metric $d$ from (10.1). Again there is a natural bijection between the points in $\mathcal{A}_{Q}\left(\mathbb{C}^{n}\right)$ and the orbits of the $\mathrm{S}_{Q}$-module $\left(\mathbb{C}^{Q}\right)^{\oplus n}$, the basic invariants of which are again given by the polarizations of the elementary symmetric functions.

Given a complex representation $G \circlearrowleft \mathbb{C}^{n}$ of a finite group $G$ we may consider the closed subspace $\mathcal{A}_{G \circlearrowleft \mathbb{C}^{n}}\left(\mathbb{C}^{n}\right)$ of $\mathcal{A}_{|G|}\left(\mathbb{C}^{n}\right)$.

The theory of complex $Q$-valued Sobolev functions can simply be taken over from the identification $\mathcal{A}_{Q}\left(\mathbb{C}^{n}\right) \cong \mathcal{A}_{Q}\left(\mathbb{R}^{2 n}\right)$ induced by $\mathbb{C} \cong \mathbb{R}^{2}$.

Let $\Omega$ be a bounded open subset of $\mathbb{R}^{m}$. With the analogous definition of the basic invariants $\sigma_{i}$ and the maps $\pi$ and $\Sigma$ we may deduce from Theorem 1.1 the following 
Theorem 10.5. Let $f: \Omega \rightarrow \mathcal{A}_{Q}\left(\mathbb{C}^{n}\right)$ be continuous. If $\Sigma \circ f \in C^{Q-1,1}\left(\bar{\Omega}, \mathbb{C}^{r}\right)$, then for each relatively compact open $\Omega^{\prime} \subseteq \Omega$ and all $1 \leq p<Q /(Q-1)$ we have $f \in W^{1, p}\left(\Omega^{\prime}, \mathcal{A}_{Q}\left(\mathbb{C}^{n}\right)\right)$. Moreover,

$$
\|D f\|_{L^{p}\left(\Omega^{\prime}\right)} \leq C\left(Q, n, m, p, \Omega, \Omega^{\prime}\right)\left(1+\max _{1 \leq j \leq r}\left\|\Sigma_{j} \circ f\right\|_{C^{Q-1,1}(\bar{\Omega})}^{1 / d_{j}}\right) .
$$

Similarly we get

Theorem 10.6. Let $f: \Omega \rightarrow \mathcal{A}_{G \circlearrowleft \mathbb{C}^{n}}\left(\mathbb{C}^{n}\right)$ be continuous. If $\Sigma \circ f \in C^{d-1,1}\left(\bar{\Omega}, \mathbb{C}^{r}\right)$, then for each relatively compact open $\Omega^{\prime} \subseteq \Omega$ and all $1 \leq p<d /(d-1)$ we have $f \in W^{1, p}\left(\Omega^{\prime}, \mathcal{A}_{G \circlearrowleft \mathbb{C}^{n}}\left(\mathbb{C}^{n}\right)\right)$. Moreover,

$$
\|D f\|_{L^{p}\left(\Omega^{\prime}\right)} \leq C\left(d, n, m, p, \Omega, \Omega^{\prime}\right)\left(1+\max _{1 \leq j \leq r}\left\|\Sigma_{j} \circ f\right\|_{C^{d-1,1}(\bar{\Omega})}^{1 / d_{j}}\right) .
$$

Again we may conclude that the bijective mapping $\Sigma$ induces a bounded mapping

$$
\left(\Sigma^{-1}\right)_{*}: C^{Q-1,1}\left(\Omega, \sigma\left(\left(\mathbb{C}^{Q}\right)^{\oplus n}\right)\right) \rightarrow W_{\mathrm{loc}}^{1, p}\left(\Omega, \mathcal{A}_{Q}\left(\mathbb{C}^{n}\right)\right), \quad \varphi \mapsto \Sigma^{-1} \circ \varphi,
$$

for all $1 \leq p<Q /(Q-1)$. In the case of a $G$-module $\mathbb{C}^{n}$ we find that

$$
\left(\Sigma^{-1}\right)_{*}: \quad C^{d-1,1}\left(\Omega, \sigma\left(\mathbb{C}^{n}\right)\right) \rightarrow W_{\mathrm{loc}}^{1, p}\left(\Omega, \mathcal{A}_{G \circlearrowleft \mathbb{C}^{n}}\left(\mathbb{C}^{n}\right)\right), \quad \varphi \mapsto \Sigma^{-1} \circ \varphi
$$

is a bounded mapping for all $1 \leq p<d /(d-1)$.

\section{Acknowledgements}

Supported by the Austrian Science Fund (FWF), Grant P 32905-N and START Programme Y963, and by ANR project ANR-17-CE40-0023 - LISA.

\section{References}

[1] Almgren Jr. F.J., Almgren's big regularity paper: $Q$-valued functions minimizing Dirichlet's integral and the regularity of area-minimizing rectifiable currents up to codimension 2, World Scientific Monograph Series in Mathematics, Vol. 1, World Sci. Publ. Co., Inc., River Edge, NJ, 2000.

[2] Chevalley C., Invariants of finite groups generated by reflections, Amer. J. Math. 77 (1955), 778-782.

[3] Dadok J., Kac V., Polar representations, J. Algebra 92 (1985), 504-524.

[4] De Lellis C., Spadaro E.N., Q-valued functions revisited, Mem. Amer. Math. Soc. 211 (2011), vi+79 pages, arXiv:0803.0060.

[5] Derksen H., Kemper G., Computational invariant theory, Encyclopaedia of Mathematical Sciences, Vol. 130, Springer-Verlag, Berlin, 2002.

[6] Ghisi M., Gobbino M., Higher order Glaeser inequalities and optimal regularity of roots of real functions, Ann. Sc. Norm. Super. Pisa Cl. Sci. (5) 12 (2013), 1001-1021, arXiv:1107.2694.

[7] Grafakos L., Classical Fourier analysis, Graduate Texts in Mathematics, Vol. 249, Springer, New York, 2014.

[8] Kriegl A., Losik M., Michor P.W., Rainer A., Lifting smooth curves over invariants for representations of compact Lie groups. III, J. Lie Theory 16 (2006), 579-600, arXiv:math.RT/0504101.

[9] Losik M., Michor P.W., Rainer A., A generalization of Puiseux's theorem and lifting curves over invariants, Rev. Mat. Complut. 25 (2012), 139-155, arXiv:0904.2068.

[10] Luna D., Slices étales, Mém. Soc. Math. France 33 (1973), 81-105.

[11] Mukai S., An introduction to invariants and moduli, Cambridge Studies in Advanced Mathematics, Vol. 81, Cambridge University Press, Cambridge, 2003.

[12] Neusel M.D., Smith L., Invariant theory of finite groups, Mathematical Surveys and Monographs, Vol. 94, Amer. Math. Soc., Providence, RI, 2002. 
[13] Parusiński A., Rainer A., A new proof of Bronshtein's theorem, J. Hyperbolic Differ. Equ. 12 (2015), 671688, arXiv:1309.2150.

[14] Parusiński A., Rainer A., Lifting differentiable curves from orbit spaces, Transform. Groups 21 (2016), 153-179, arXiv:1406.2485.

[15] Parusiński A., Rainer A., Regularity of roots of polynomials, Ann. Sc. Norm. Super. Pisa Cl. Sci. (5) 16 (2016), 481-517, arXiv:1309.2151.

[16] Parusiński A., Rainer A., Optimal Sobolev regularity of roots of polynomials, Ann. Sci. Éc. Norm. Supér. (4) 51 (2018), 1343-1387, arXiv:1506.01512.

[17] Parusiński A., Rainer A., Selections of bounded variation for roots of smooth polynomials, Selecta Math. (N.S.) 26 (2020), 13, 40 pages, arXiv:1705.10492.

[18] Schwarz G.W., Lifting smooth homotopies of orbit spaces, Inst. Hautes Études Sci. Publ. Math. 51 (1980), 37-135.

[19] Serre J.-P., Groupes finis d'automorphismes d'anneaux locaux réguliers, in Colloque d'Algèbre (Paris, 1967), Secrétariat mathématique, Paris, 1968, Exp. 8, 11 pages.

[20] Shephard G.C., Todd J.A., Finite unitary reflection groups, Canad. J. Math. 6 (1954), 274-304.

[21] Weyl H., The classical groups. Their invariants and representations, Princeton University Press, Princeton, N.J., 1939. 